MATHEMATICS OF COMPUTATION

Volume 76, Number 260, October 2007, Pages 1955-1980

S 0025-5718(07)01937-0

Article electronically published on April 27, 2007

\title{
THE CONSTRUCTION OF CUBATURE RULES FOR MULTIVARIATE HIGHLY OSCILLATORY INTEGRALS
}

\author{
DAAN HUYBRECHS AND STEFAN VANDEWALLE
}

\begin{abstract}
We present an efficient approach to evaluate multivariate highly oscillatory integrals on piecewise analytic integration domains. Cubature rules are developed that only require the evaluation of the integrand and its derivatives in a limited set of points. A general method is presented to identify these points and to compute the weights of the corresponding rule.

The accuracy of the constructed rules increases with increasing frequency of the integrand. For a fixed frequency, the accuracy can be improved by incorporating more derivatives of the integrand. The results are illustrated numerically for Fourier integrals on a circle and on the unit ball, and for more general oscillators on a rectangular domain.
\end{abstract}

\section{INTRODUCTION}

Oscillatory integrals frequently arise in computational methods for oscillatory phenomena in science and engineering, including wave scattering, wave propagation and quantum mechanics. Their efficient evaluation is an important step in the construction of accurate solution methods for these types of problems. In this paper, we present the construction of a family of cubature rules for multivariate oscillatory integrals, that require only few weights and abscissae. These rules yield an approximation to the value of the integral with an accuracy that increases with increasing frequency of the oscillations. The abscissae depend only on the type of oscillator in the integral; the weights depend also on the frequency.

We consider an $n$-dimensional oscillatory integral with a general form given by

$$
I_{n}:=\int_{S} f(\mathbf{x}) e^{i \omega g(\mathbf{x})} \mathrm{d} \mathbf{x}
$$

where $S$ represents an integration domain that can be described by a piecewise analytic parameterization. The function $g$ is called the oscillator of (1.1). Intuitively, it is clear that the value of $I_{n}$ will decrease as the frequency parameter $\omega$ increases, because the oscillations of the integrand increasingly cancel out. We will show that the value of $I_{n}$ is determined largely by the behaviour of $f$ and $g$ near a set of so-called critical points. These points correspond to the points where the integrand locally does not oscillate, or to certain points on the boundary. The former set are the points where the gradient of $g$ vanishes, the so-called stationary

Received by the editor November 21, 2005 and, in revised form, April 12, 2006.

2000 Mathematics Subject Classification. Primary 65D32; Secondary 41A60, 41A63.

Key words and phrases. Cubature formulas, oscillatory functions, steepest descent.

The first author was supported by The Institute for the Promotion of Innovation through Science and Technology in Flanders (IWT-Vlaanderen).

(C)2007 American Mathematical Society Reverts to public domain 28 years from publication 
points; the latter set contains points where the gradient of $g$ is orthogonal to the boundary, and the corner points of the integration domain.

There exist several approaches for the evaluation of one-dimensional oscillatory integrals $I_{1}$ with a similar form. An overview of available methods can be found in [6] and in the references therein. Here, we recall a number of approaches that become increasingly effective with increasing frequency, and that can be extended to a multivariate setting. They are asymptotic methods, Filon-type methods and Levin-type methods.

For large values of $\omega$, the value of the multivariate definite integral $I_{n}$ can be approximated by an asymptotic expansion of the integral 1, 14, 15. The stationary points are usually assumed to be nondegenerate, i.e., it is assumed that the Hessian at the point is not singular. A disadvantage of asymptotic expansions is that, in most cases, it is not straightforward to obtain the coefficients of the expansion. A different asymptotic expansion was constructed for univariate oscillatory integrals in [7, 8], with explicit expressions for the coefficients in terms of the derivatives of $f$ and $g$ at the critical points. This approach was extended to multivariate integrals without stationary points on polytope domains, and to a number of double integrals involving nondegenerate stationary points in 9,10 . In the same references, Filon's method for oscillatory integrals was generalised and extended to these cases. The resulting Filon-type methods lead to a quadrature rule or cubature rule involving function values and derivatives of $f$ at a small number of points. We will revisit Filon-type methods in $\$ 2.3$.

A different approach was taken in [11, by associating the oscillatory integral with a system of ordinary differential equations that is solved by collocation. This method was extended to yield high order accuracy as a function of $\omega$ for univariate integrals and multivariate integrals in 12, 13, subject to a nonresonance condition that excludes stationary points and points where the gradient of the oscillator is orthogonal to the boundary. These so-called Levin-type methods do not require the computation of weights or moments.

In this paper, we present a new type of cubature rules for integrals of the form (1.1). The method works in the presence of nondegenerate or degenerate stationary points, and also for any shape of the integration domain. It is shown that the weights can be evaluated efficiently. The convergence of the rules is analysed and illustrated with some examples. We start in $\$ 2$ with a review of the one-dimensional approach of [6]. We extend the approach to a number of two-

dimensional integrals by some motivating examples in 83 . The general theory for multivariate integrals is given in $\$$. The construction of cubature rules is discussed in 95 . Some numerical experiments illustrate the approach in 96 . Finally, we end with some concluding remarks in $\$ 7$

\section{RevieW OF THE ONE DIMENSIONAL CASE}

In this section we recall the main results of [6]. As a model for one-dimensional oscillatory integrals, we consider the integral

$$
I_{1}:=\int_{a}^{b} f(x) e^{i \omega g(x)} \mathrm{d} x
$$


with $i=\sqrt{-1}$ the imaginary root, and with $f$ and $g$ smooth functions. We call $g(x)$ the oscillator of (2.1). We will first discuss the case without stationary points, i.e., we assume that the equation $g^{\prime}(x)=0$ has no solution on $[a, b]$.

2.1. The ideal case without stationary points. If both $f$ and $g$ are analytic, then the integrand of (2.1) is also analytic. In that case, we may choose a different integration path from $a$ to $b$ in the complex plane by Cauchy's Theorem [5]. A useful observation is that the function $e^{i \omega g(x)}$ oscillates only if the real part of $g(x)$ varies. The function decays exponentially fast with an increasing imaginary part, since $e^{i \omega(a+b i)}=e^{-\omega b} e^{i \omega a}$. With this observation in mind, an integration path can be found for (2.1) that goes from $a$ to a region of the complex plane where the integrand becomes arbitrarily small, and from that region back to the point $b$. First, we recall an important theoretical result from [6].

Theorem 2.1 ([6], Th 3.2). Assume $f$ and $g$ are analytic in a bounded and open complex neighbourhood $D$ of $[a, b]$, and $g^{\prime}(z) \neq 0, z \in D$. Then there exists a function $F(x), x \in[a, b]$, such that

$$
\int_{a}^{x} f(z) e^{i \omega g(z)} \mathrm{d} z=F(a)-F(x)+O\left(e^{-\omega d_{0}}\right),
$$

with $d_{0}>0$ and where $F(x)$ is of the form

$$
F(x)=\int_{\Gamma_{x}} u(z) \mathrm{d} z
$$

with $\Gamma_{x}$ a path that starts at $x$, and with an exponentially decaying integrand along that path.

Assume that the path $\Gamma_{x}$ in the definition of $F$ is parameterized by $h_{x}(p), p \in$ $[0, P]$. A particularly useful choice for $h_{x}(p)$ satisfies

$$
g\left(h_{x}(p)\right)=g(x)+i p
$$

This path is called the path of steepest descent [1, 15. Expression (2.3) for $F(x)$ can then be written as

$$
F(x)=\int_{0}^{P} f\left(h_{x}(p)\right) e^{i \omega g\left(h_{x}(p)\right)} h_{x}^{\prime}(p) \mathrm{d} p=e^{i \omega g(x)} \int_{0}^{P} f\left(h_{x}(p)\right) h_{x}^{\prime}(p) e^{-\omega p} \mathrm{~d} p .
$$

The resulting integrand does not oscillate and decays exponentially fast. In the limiting case $P \rightarrow \infty$, approximation (2.2) becomes exact, i.e., the term $O\left(e^{-\omega d_{0}}\right)$ vanishes, for all values of $\omega$ greater than a lower bound $\omega_{0}$ [6, Th 3.3]. The function $F(x)$ can then be evaluated efficiently by Gauss-Laguerre integration [2]. The convergence increases rapidly as the frequency parameter $\omega$ increases, as shown in the following theorem.

Theorem 2.2 ([ $[$, Th 3.5). Let $F(x)$ be approximated by the quadrature formula

$$
F(x) \approx Q_{F}\left[f, g, h_{x}\right]:=\frac{e^{i \omega g(x)}}{\omega} \sum_{i=1}^{n} w_{i} f\left(h_{x}\left(x_{i} / \omega\right)\right) h_{x}^{\prime}\left(x_{i} / \omega\right),
$$

with $w_{i}$ and $x_{i}$ the weights and abscissae of an n-point Gauss-Laguerre quadrature rule. Then the quadrature error behaves asymptotically as $O\left(\omega^{-2 n-1}\right)$. 
For complex functions $g$, the equation $g\left(h_{x}(p)\right)=g(x)+i p$ may not be easily solvable. The function $F(x)$ may still be evaluated by approximating the optimal path $h_{x}(p)$ locally around $x$ using, e.g., a Taylor series of $g$ with only few terms. A straightforward Newton-Raphson iteration process can additionally be applied to yield the exact optimal path. Finally, an approximation of $F(x)$ can be obtained by replacing $g$ by its truncated Taylor series in the definition of $F$. Convergence estimates for this approach are derived in 6 . We note that the implementation of this scheme is entirely numerical, whereas the method of Steepest Descent usually leads to an asymptotic expansion based on analytic manipulations of the integrand.

2.2. Stationary points. A stationary point is any solution $\xi$ to the equation $g^{\prime}(x)=0$. The importance of stationary points lies in the fact that the integrand of (2.1) locally does not oscillate near $\xi$; the presence of stationary points therefore has a large effect on the value of $I_{1}$.

The inverse of $g$ is multi-valued in a neighbourhood of any stationary point. Equation (2.4), that was used to find the optimal path, has at least two possible solutions for $h_{x}(p)$. The ambiguity is resolved by restricting the integration interval $[a, b]$ to the subintervals $[a, \xi]$ and $[\xi, b]$ respectively. On each subinterval, a singlevalued analytic branch of the inverse of $g$ exists, that can be used to solve (2.4). The results of Theorem 2.1 are extended in the following theorem.

Theorem 2.3 ([6], Th 4.1). Assume that the functions $f$ and $g$ are analytic in a bounded and open complex neighbourhood $D$ of $[a, b]$. If the equation $g^{\prime}(x)=0$ has only one solution $\xi$ in $D$ and $\xi \in(a, b)$, then there exist functions $F_{j}(x), j=1,2$, such that

$$
\int_{s}^{t} f(z) e^{i \omega g(z)} \mathrm{d} z=F_{1}(s)-F_{1}(\xi)+F_{2}(\xi)-F_{2}(t)+O\left(e^{-\omega d_{0}}\right), \quad d_{0}>0,
$$

for $s \in[a, \xi]$ and $t \in[\xi, b]$, where $F_{j}(x)$ is of the form

$$
F_{j}(x):=\int_{\Gamma_{x, j}} f(z) e^{i \omega g(z)} \mathrm{d} z
$$

with $\Gamma_{x, j}$ a path that starts at $x$, and with an exponentially decaying integrand along that path.

Similarly, a decomposition can be found for the case of multiple stationary points. Assume there are $l$ stationary points $\xi_{i} \in(a, b), i=1, \ldots, l$. Define $\xi_{0}:=a$ and $\xi_{l+1}:=b$. Then Theorem 2.3 can be applied repeatedly to yield a decomposition of the form

$$
I_{1} \approx \sum_{i=1}^{l+1} F_{i}\left(\xi_{i-1}\right)-F_{i}\left(\xi_{i}\right) .
$$

Unfortunately, the parameterization $h_{\xi, j}(p)$ for $\Gamma_{\xi, j}$ exhibits a numerical problem at $p=0$. Define $r$ as the smallest integer such that $g^{(r+1)}(\xi) \neq 0$, i.e., all derivatives of $g$ vanish at $\xi$ up to order $r$. We say that the stationary point has order $r$. Then the derivative of the optimal path, $h_{\xi, j}^{\prime}(p)$, behaves as $O\left(p^{-r /(r+1)}\right), p \rightarrow 0$. This singularity prevents convergence of the Gauss-Laguerre quadrature approach that was used in 22.1 . However, it can be dealt with efficiently by generalized GaussLaguerre quadrature [2]. Generalized Gauss-Laguerre polynomials are orthogonal with respect to the weight function $x^{\alpha} e^{-x}, \alpha>-1$. The corresponding Gaussian quadrature rules can be applied with the choice $\alpha=-r /(r+1)$. 
Theorem $2.4(\underline{6}]$, Th 4.4). Assume that $g^{(k)}(\xi)=0, k=1, \ldots, r$, and $g^{(r+1)}(\xi) \neq$ 0 . Let the function $F_{j}(\xi)$ be approximated by the quadrature formula

$$
F_{j}(\xi) \approx Q_{F}^{\alpha}\left[f, g, h_{\xi, j}\right]:=\frac{e^{i \omega g(\xi)}}{\omega} \sum_{i=1}^{n} w_{i} f\left(h_{\xi, j}\left(x_{i} / \omega\right)\right) h_{\xi, j}^{\prime}\left(x_{i} / \omega\right) x_{i}^{-\alpha},
$$

with $w_{i}$ and $x_{i}$ the weights and abscissae of an n-point generalized Gauss-Laguerre quadrature rule with $\alpha=-r /(r+1)$. Then the error behaves asymptotically as $O\left(\omega^{-2 n-1 /(r+1)}\right)$.

The relative error of both the approximation of $F(x)$ in Theorem 2.2 and the approximation of $F_{j}(\xi)$ in Theorem [2.4] scales as $O\left(\omega^{-2 n}\right)$.

2.3. Quadrature rules using derivatives. For large values of the frequency parameter $\omega$, the value of $F_{j}(x)$ depends only on the behaviour of $f$ and $g$ around the point $x$. It can be seen from the quadrature rules (2.5) and (2.9) that the path and its derivative are only evaluated near $p=0$. This is a general observation: the value of $I_{1}$ depends only on the behaviour of $f$ and $g$ around the boundary points $a$ and $b$, and the stationary points $\xi$ in the integration interval. Assume that $f$ can be approximated well around these points by a linear combination of certain basis functions, i.e., $f=\sum_{i=1}^{N} a_{i} \phi_{i}(x)$. The integral of these basis functions, the so-called moments, can then be used as the weights of a quadrature rule to evaluate (2.1),

$$
I_{1} \approx \sum_{i=1}^{N} a_{i} w_{i} \quad \text { with } \quad w_{i}:=\int_{a}^{b} \phi_{i}(x) e^{i \omega g(x)} \mathrm{d} x .
$$

This is essentially a description of Filon's method for oscillatory integrals 3 . This method was extended by Iserles and Nørsett in 7 to yield higher order accuracy as a function of $1 / \omega$. These authors proposed the use of a polynomial basis and Hermite interpolation of $f$. The interpolation of $f$ and a number of derivatives at $a, b$ and any stationary point in between, yields an accuracy of order $O\left(\omega^{-s-1}\right)$, where $s$ depends on the number of interpolated derivatives and on the order of the stationary points. The quadrature rule requires evaluating $f$ and its derivatives at the interpolation points. The weights of the rule can be computed for general oscillators $g$ using the approach from [6].

Owing to the decomposition of $I_{1}$ in a sum of terms that can be evaluated independently from each other, it becomes possible to approximate $f$ by different polynomials locally around each of the critical points. This localised approach allows the use of a much lower degree of approximation at each critical point. Define the moments $\mu_{j, k}(x)$ as

$$
\mu_{j, k}(x):=\int_{\Gamma_{x, j}}(z-x)^{k} e^{i \omega g(z)} \mathrm{d} z
$$

with $\Gamma_{x, j}$ the same curve as in the definition (2.7) of $F_{j}$; then we have

$$
F_{j}(x)=\sum_{k=0}^{\infty} f^{(k)}(x) \frac{\mu_{j, k}(x)}{k !} .
$$

The convergence rate of this series depends on the size of the coefficients. It was proven in [6] that $\left|\mu_{j, k}(x)\right|=O\left(\omega^{-k-1}\right)$ for regular points, and $\left|\mu_{j, k}(\xi)\right|=$ $O\left(\omega^{(-k-1) /(r+1)}\right)$ for stationary points. For a fixed $g$ and a fixed interval $[a, b]$, 
substituting (2.12) into the decomposition (2.8) yields a quadrature rule for the value of $I_{1}$ that uses derivatives of $f$,

$$
I_{1} \approx Q[f]:=\sum_{j=0}^{l+1} \sum_{k=0}^{r_{j}} w_{j, k} f^{(k)}\left(\xi_{j}\right) .
$$

The weights are given by $w_{0, k}:=\frac{\mu_{0, k}(a)}{k !}, w_{l+1, k}:=-\frac{\mu_{l, k}(b)}{k !}$ and $w_{j, k}:=\frac{\mu_{j, k}\left(\xi_{j}\right)}{k !}-$ $\frac{\mu_{j-1, k}\left(\xi_{j}\right)}{k !}, j=1, \ldots, l$. The asymptotic behaviour of the error of the quadrature is given by the behaviour of the first term of expansion (2.12) that is discarded. The number of derivatives that are used at each special point $\xi_{j}$, given by the constants $r_{j}$ in (2.13), can be varied such that the order of the truncation error is the same at each point. In particular, the number of derivatives needs to be larger at stationary points.

\section{EXTENSION TO TWO-DIMENSIONAL INTEGRALS}

In this section, we extend the results of the one-dimensional approach to twodimensional oscillatory integrals. The problems that arise are introduced one by one, in a series of examples that become exceedingly more general. First, we consider the integration on a rectangular domain which will be handled by repeated one-dimensional integration. Next, we generalize to smooth integration boundaries. This generalization leads to a number of points that are similar to stationary points in one dimension. Finally, we study an example with points internal to the domain where the gradient function $\nabla g$ vanishes. Such points appear as stationary points in each integration variable.

In this section, we will assume that all considered functions $f$ and $g$ are such that the error in the decompositions of Theorems 2.1 and 2.3 vanishes. Sufficient conditions for $f$ and $g$ that enable this limit case are given in [6]; they involve conditions on the growth of $f$ and of the inverse of $g$ in the complex plane. This assumption is made in this section purely for the sake of clarity and brevity. The theory will be described without this assumption in Section 4 .

3.1. Rectangular domains in two dimensions. The simplest extension of the one-dimensional method to multivariate integrals is the use of repeated one-dimensional integration on a rectangular domain. In order to illustrate the basic approach, we restrict the discussion to a strictly monotonically increasing function $g$. Therefore consider the double integral

$$
I_{2}:=\int_{a}^{b} \int_{c}^{d} f(x, y) e^{i \omega(x+y)} \mathrm{d} y \mathrm{~d} x
$$

with $f$ analytic in both variables $x$ and $y$. For a fixed value of $x$, the inner integration in $y$ can be written as a finite sum of contributions by applying Theorem 2.1. We have

$$
\int_{c}^{d} f(x, y) e^{i \omega(x+y)} \mathrm{d} y=G(x, c)-G(x, d) .
$$

An expression for $G(x, y)$ is given by

$$
G(x, y)=e^{i \omega(x+y)} \int_{0}^{\infty} f\left(x, v_{y}(x, q)\right) \frac{\partial v_{y}}{\partial q}(x, q) e^{-\omega q} \mathrm{~d} q
$$


where $v_{y}(x, q)$ is found as the solution to $g\left(x, v_{y}(x, q)\right)=g(x, y)+q i$. The particular oscillator $g(x, y)=x+y$ in this example leads to the path $v_{y}(x, q):=y+q i$. An important observation is that the function $G(x, y)$ is analytic as a function of $x$, because all factors in expression (3.2) are analytic in $x$. In addition, $G(x, y)$ is an oscillatory function of $x$ with the oscillator $g_{1}(x):=x$. Hence, the integration of $G(x, y)$ in $x$ can also be written as a sum of contributions. The optimal path is given by $u_{x}(p):=x+i p$. We arrive at

$$
I_{2}=\int_{a}^{b}(G(x, c)-G(x, d)) \mathrm{d} x=[F(a, c)-F(b, c)]-[F(a, d)-F(b, d)],
$$

where the function $F(x, y)$ is given by

$$
\begin{aligned}
F(x, y) & =e^{i \omega(x+y)} \int_{0}^{\infty} \int_{0}^{\infty} f\left(u_{x}(p), v_{y}\left(u_{x}(p), q\right)\right) u_{x}^{\prime}(p) \frac{\partial v_{y}}{\partial q}\left(u_{x}(p), q\right) e^{-\omega(p+q)} \mathrm{d} q \mathrm{~d} p \\
& =e^{i \omega(x+y)} \int_{0}^{\infty} \int_{0}^{\infty} f(x+i p, y+q i) i^{2} e^{-\omega(p+q)} \mathrm{d} q \mathrm{~d} p .
\end{aligned}
$$

The value of $I_{2}$ is found by summing the contributions from each of the corner points of the rectangular domain. These contributions are given by a double integral with a nonoscillating integrand that decays exponentially fast as a function of both integration variables. They can be evaluated efficiently using, e.g., tensor-product Gauss-Laguerre quadrature.

3.2. Smooth boundaries in two dimensions. The double integral (3.1) is generalized by considering integration boundaries for $y$ that depend on $x$. The simplest of those extensions is a simplex. We therefore first consider the evaluation of the following integral:

$$
I_{2}:=\int_{a}^{b} \int_{a}^{x} f(x, y) e^{i \omega(x+y)} \mathrm{d} y \mathrm{~d} x .
$$

Applying Theorem 2.1 for the inner integration in $y$ leads to

$$
\int_{a}^{x} f(x, y) e^{i \omega(x+y)} \mathrm{d} y=G(x, a)-G(x, x),
$$

with $G(x, y)$ again given by (3.2). The term $G(x, x)$ did not appear before; it is given by

$$
G(x, x)=e^{i \omega 2 x} \int_{0}^{\infty} f(x, x+q i) i e^{-\omega q} \mathrm{~d} q .
$$

This means that the oscillators in $x$ of $G(x, a)$ and of $G(x, x)$ are different: they are respectively given by $g_{1}(x):=x$ and $g_{2}(x):=2 x$. A decomposition can be found for the integration in $x$, applying Theorem 2.1 for both terms separately. This yields

$$
I_{2}=\int_{a}^{b}(G(x, a)-G(x, x)) \mathrm{d} x=\left[F_{1}(a, a)-F_{1}(b, a)\right]-\left[F_{2}(a, a)-F_{2}(b, b)\right]
$$

with $F_{1}(x, y):=F(x, y)$ corresponding to the integral of $G(x, a)$, and $F_{2}$ given by

$$
F_{2}(x, x)=e^{i \omega 2 x} \int_{0}^{\infty} \int_{0}^{\infty} f\left(x+\frac{p}{2} i, x+\frac{p}{2} i+q i\right) \frac{i^{2}}{2} e^{-\omega(p+q)} \mathrm{d} q \mathrm{~d} p .
$$

This expression is obtained by following the paths $v_{y}(x, q)=y+q i$ and $u_{x}(p)=$ $x+\frac{p}{2} i$. Although function $F_{2}$ is a function of only one variable $x$, the notation $F_{2}(x, x)$ is used for later notational convenience. Note that all contributions in the 
total decomposition are given by the evaluation of a function $F_{1}$ or $F_{2}$ at a corner point of the simplex.

A new difficulty arises when the boundaries of the integration in $y$ are more general. Assume analytic functions $c(x)$ and $d(x)$ are given and define the integral

$$
I_{2}:=\int_{a}^{b} \int_{c(x)}^{d(x)} f(x, y) e^{i \omega(x+y)} \mathrm{d} y \mathrm{~d} x .
$$

Decomposing the inner integration in $y$ now leads to

$$
\begin{gathered}
\int_{c(x)}^{d(x)} f(x, y) e^{i \omega(x+y)} \mathrm{d} y=G(x, c(x))-G(x, d(x)) \\
=e^{i \omega(x+c(x))} \int_{0}^{\infty} f(x, c(x)+q i) i e^{-\omega q} \mathrm{~d} q \\
-e^{i \omega(x+d(x))} \int_{0}^{\infty} f(x, d(x)+q i) i e^{-\omega q} \mathrm{~d} q .
\end{gathered}
$$

The oscillator of $G(x, c(x))$ is $g_{1}(x):=g(x, c(x))=x+c(x)$. Although the partial derivatives of the original function $g(x, y)=x+y$ do not vanish anywhere, the function $g_{1}(x)$ may have stationary points:

$$
\frac{\mathrm{d}}{\mathrm{d} x} g(x, c(x))=\frac{\partial g}{\partial x}+\frac{\partial g}{\partial y} \frac{\mathrm{d} c}{\mathrm{~d} x}=0 \Longleftrightarrow \nabla g \cdot \nabla\left[\begin{array}{c}
x \\
c(x)
\end{array}\right]=0 .
$$

Function $g_{1}(x)$ has a stationary point in $x$ if the gradient of $g(x, y)$ is orthogonal to the tangent line of the boundary, which is parameterized by $[x c(x)]^{T}$. This condition was already identified by Iserles and Nørsett in [9]. There, the case was explicitly excluded by the so-called nonresonance condition. Clearly, this is a severe restriction, since it occurs easily for smooth boundaries.

Assume that $g_{1}(x)$ has $l_{c}$ stationary points $\xi_{c, i} \in(a, b), i=1, \ldots, l_{c}$, and $g_{2}(x):=$ $g(x, d(x))=x+d(x)$ has $l_{d}$ stationary points $\xi_{d, i} \in(a, b), i=1, \ldots, l_{d}$. Set $\xi_{c, 0}:=a$, $\xi_{c, l_{c}+1}:=b, \xi_{d, 0}:=a$ and $\xi_{d, l_{d}+1}:=b$. Then we can use the shorthand notation of (2.8) to write $I_{2}$ as

$$
\begin{aligned}
I_{2}= & \sum_{i=1}^{l_{c}+1}\left[F_{1, i}\left(\xi_{c, i-1}, c\left(\xi_{c, i-1}\right)\right)-F_{1, i}\left(\xi_{c, i}, c\left(\xi_{c, i}\right)\right)\right] \\
& -\sum_{i=1}^{l_{d}+1}\left[F_{2, i}\left(\xi_{d, i-1}, d\left(\xi_{d, i-1}\right)\right)-F_{2, i}\left(\xi_{d, i}, d\left(\xi_{d, i}\right)\right)\right] .
\end{aligned}
$$

The contributions come from the boundary points $(a, c(a)),(b, c(b)),(a, d(a))$ and $(b, d(b))$, and also from other points on the boundary, given by $\left(\xi_{c, i}, c\left(\xi_{c, i}\right)\right)$ and $\left(\xi_{d, i}, d\left(\xi_{d, i}\right)\right)$. The latter are all the points where the gradient of $g$ is orthogonal to the boundary.

Note that for a simplex we have $c(x)=a$ and $d(x)=x$. For the particular choice of oscillator $g(x, y):=x-y$, we have $g_{2}(x):=g(x, d(x))=0$. In other words, the function $G(x, d(x))$ is not oscillatory at all! The gradient of $g$ is orthogonal to the boundary at all points $(x, x)$. For this particular case, the integration in $x$ cannot be written as a sum of contributions. However, there is no need for a decomposition, as the integral $\int_{a}^{b} G(x, d(x)) \mathrm{d} x$ can be evaluated by, e.g., regular Gaussian quadrature on the real line $[a, b]$. Note that this case is exceptional, while the condition that the gradient of $g$ is orthogonal to the boundary in only one point is common. 
3.3. Stationary points. A final complication that may arise in decomposing highly oscillatory two-dimensional integrals into a sum of contributions, is the presence of stationary points where $\nabla g=0$. Consider the model integral

$$
I_{2}:=\int_{a}^{b} \int_{c}^{d} f(x, y) e^{i \omega\left(x^{2}-x y-y^{2}\right)} \mathrm{d} y \mathrm{~d} x
$$

with $a, c<0$ and $b, d>0$. We have $g(x, y)=x^{2}-x y-y^{2}$ and $\nabla g(0,0)=0$ in the internal point $(0,0)$. In the following we will derive a decomposition for $I_{2}$ as a sum of contributions of the form $F_{j k l}(x, y)$. Each function $F_{j k l}$ is evaluated in a special point that is to be determined. The index $j$ denotes the path for $y: v_{y, j}(x, q)$. The combination of index $j$ and index $k$ denotes the different oscillators in $x$ that result: $g_{j k}(x)$. Finally, index $l$ is used to denote the path for $x: u_{x, j k l}(p)$. The general form of the contribution $F_{j k l}$ will be shown to be

$$
\begin{array}{r}
F_{j k l}(x, y)=e^{i \omega g_{j k}(x)} \int_{0}^{\infty} \int_{0}^{\infty} f\left(u_{x, j k l}(p), v_{y, j}\left(u_{x, j k l}(p), q\right)\right) \\
\frac{\partial u_{x, j k l}}{\partial p}(p) \frac{\partial v_{y, j}}{\partial q}\left(u_{x, j k l}(p), q\right) e^{-\omega(p+q)} \mathrm{d} q \mathrm{~d} p .
\end{array}
$$

3.3.1. Stationary points in $y$. For any $x \in[a, b]$, function $g(x, y)$ has a stationary point in $y$ given by $y=-x / 2$, since $\frac{\partial g}{\partial y}(x,-x / 2)=0$. We can write the integral (3.6) as

$$
\begin{aligned}
\int_{c}^{d} f(x, y) e^{i \omega g(x, y)} \mathrm{d} y & =\int_{c}^{-x / 2} f(x, y) e^{i \omega\left(x^{2}-x y-y^{2}\right)} \mathrm{d} y \\
& +\int_{-x / 2}^{d} f(x, y) e^{i \omega\left(x^{2}-x y-y^{2}\right)} \mathrm{d} y .
\end{aligned}
$$

For this decomposition, we have assumed that $c \leq-b / 2$ and $-a / 2 \leq d$, as illustrated in Figure 1. The problem has now become similar to the problem of a smooth boundary treated earlier. Consider the first integral in the right hand side of (3.8). By Theorem 2.3, there exists a decomposition

$$
\int_{c}^{-x / 2} f(x, y) e^{i \omega\left(x^{2}-x y-y^{2}\right)} \mathrm{d} y=G_{1}(x, c)-G_{1}(x,-x / 2) .
$$

The path for $y$ is found by solving $g\left(x, v_{y, 1}(x, q)\right)=g(x, y)+q i$, and leads to

$$
v_{y, 1}(x, q)=-x / 2-1 / 2 \sqrt{x^{2}+4 x y+4 y^{2}-4 i q} .
$$

The function $G_{1}(x, y)$ is given in its general form by

$$
G_{1}(x, y)=e^{i \omega g(x, y)} \int_{0}^{\infty} f\left(x, v_{y, 1}(x, q)\right) \frac{\partial v_{y, 1}}{\partial q}(x, q) e^{-\omega q} \mathrm{~d} q,
$$

and thus $G_{1}(x, c)$ has an oscillator $g_{11}(x):=x^{2}-c x-c^{2}$, with a stationary point at $x=c / 2$. The latter corresponds to the point $(c / 2, c)$ on the integration boundary. The oscillator for $G_{1}(x,-x / 2)$ is $g_{12}(x):=5 / 4 x^{2}$, with a stationary point at $x=0$. This corresponds to the internal point $(0,0)$.

Similarly, the second integral in the right hand side of (3.8) can be written as

$$
\int_{-x / 2}^{d} f(x, y) e^{i \omega\left(x^{2}-x y-y^{2}\right)} \mathrm{d} y=G_{2}(x,-x / 2)-G_{2}(x, d) .
$$




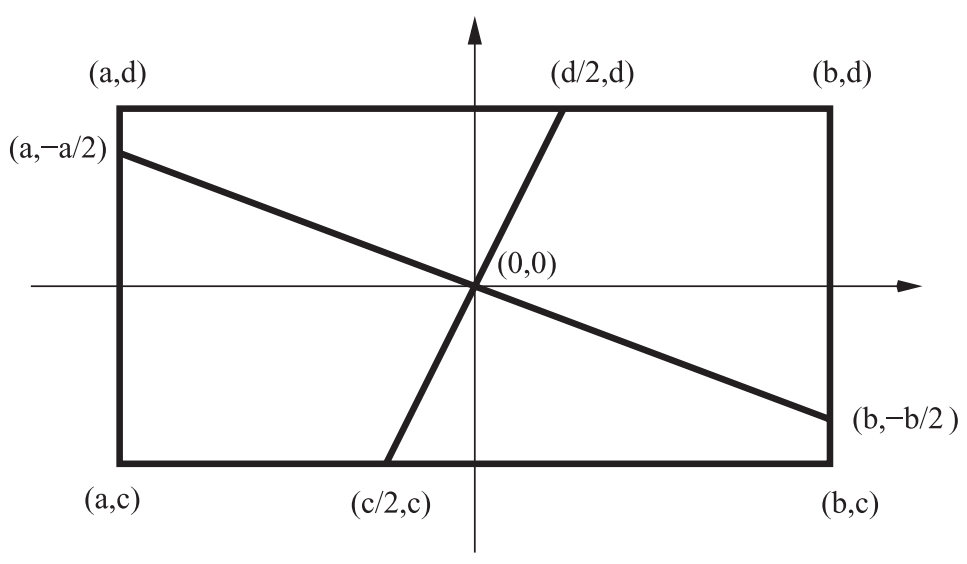

Figure 1. The points that contribute to the double integral $I_{2}$ for $g(x, y)=x^{2}-x y-y^{2}$ on the rectangle $[a, b] \times[c, d]$.

The path for $y$ differs from the path used to obtain the function $G_{1}$. We denote the path by $v_{y, 2}(x, q)$, and note that it is given by

$$
v_{y, 2}(x, q)=-x / 2+1 / 2 \sqrt{x^{2}+4 x y+4 y^{2}-4 i q} .
$$

We can define oscillators $g_{21}(x):=5 / 4 x^{2}$ and $g_{22}(x):=x^{2}-d x-d^{2}$, corresponding to the functions $G_{2}(x,-x / 2)$ and $G_{2}(x, d)$ respectively. They have a stationary point at $x=0$ and $x=d / 2$.

3.3.2. Stationary points in $x$. We have already shown that $I_{2}$ can be written as a sum of four integrals of the form

$$
\int_{a}^{b} G_{j}\left(x, s_{j k}(x)\right) \mathrm{d} x, \quad j=1,2, \quad k=1,2,
$$

where each $G_{j}\left(x, s_{j k}(x)\right)$ has an oscillator in $x$ of the form $g_{j k}(x):=g\left(x, s_{j k}(x)\right)$, with one stationary point $x=\xi_{j k}$. Applying Theorem 2.3 shows the existence of two functions $F_{j k 1}(x, y)$ and $F_{j k 2}(x, y)$ such that

$$
\begin{aligned}
\int_{a}^{b} G_{j}\left(x, s_{j k}(x)\right) \mathrm{d} x= & F_{j k 1}\left(a, s_{j k}(a)\right)-F_{j k 1}\left(\xi_{j k}, s_{j k}\left(\xi_{j k}\right)\right) \\
& +F_{j k 2}\left(\xi_{j k}, s_{j k}\left(\xi_{j k}\right)\right)-F_{j k 2}\left(b, s_{j k}(b)\right) .
\end{aligned}
$$

The paths for $x$ are found by solving $g_{j k}\left(u_{j k l}(p)\right)=g_{j k}\left(x_{j k l}\right)+i p, j=1,2, k=$ $1,2, l=1,2$. Analytic expressions are easily derived: for the oscillator $g_{21}(x)=$ $g(x,-x / 2)=5 / 4 x^{2}$, evaluated at $x_{211}=\xi_{21}=0$, we find

$$
g_{21}\left(u_{211}(p)\right)=g_{21}\left(x_{211}\right)+i p \Rightarrow \frac{5}{4} u_{211}^{2}(p)=i p \Rightarrow u_{211}(p)=\sqrt{\frac{4}{5} i p} .
$$

We have arrived at a decomposition for $I_{2}$ with 16 functions of the form (3.7). Substituting the functions $s_{11}(x)=c, s_{12}(x)=s_{21}(x)=-x / 2$ and $s_{22}(x)=d$ into 
the general form, the total decomposition is given by

$$
\begin{aligned}
I_{2} & =F_{111}(a, c)-F_{111}(c / 2, c)+F_{112}(c / 2, c)-F_{112}(b, c) \\
& -F_{121}(a,-a / 2)+F_{121}(0,0)-F_{122}(0,0)+F_{122}(b,-b / 2) \\
& +F_{211}(a,-a / 2)-F_{211}(0,0)+F_{212}(0,0)-F_{212}(b,-b / 2) \\
& -F_{221}(a, d)+F_{221}(d / 2, d)-F_{222}(d / 2, d)+F_{222}(b, d) .
\end{aligned}
$$

There is one evaluation in each corner point, there are two evaluations in the points where $\nabla g$ is orthogonal to the boundary, and there are four evaluations in the central stationary point $(0,0)$ where $\nabla g$ vanishes in all integration variables. All relevant points are shown in Figure1. The lines connecting $(a,-a / 2)$ with $(b,-b / 2)$, and $(c / 2, c)$ with $(d / 2, d)$ are given by $y=-x / 2$ and $x=y / 2$ respectively: they correspond to curves along which the partial derivative of $g(x, y)$ with respect to $x$ or $y$ vanishes. They intersect in the stationary point.

\section{A DECOMPOSition OF MUltivariate Highly OSCILlatory INTEGRAlS}

In the previous section, we have illustrated the issues that arise in identifying the individual contributions to oscillatory integrals in two dimensions. These examples will motivate and clarify the results for the general $n$-dimensional case. First, we prove a decomposition for a one-dimensional integral of an $n$-dimensional function in 4.1 . Next, a decomposition of multivariate integrals is obtained by repeated one-dimensional integration in 4.2 .

4.1. A decomposition for one variable. The decomposition of a one-dimensional integral is given in Theorem 2.1 for the case without stationary points, and in Theorem 2.3 in the presence of a stationary point. Here, we will refine Theorem 2.1 and obtain an expression for the error of the decomposition.

Lemma 4.1. Assume that the functions $f$ and $g$ are analytic in an open complex neighbourhood $D$ of $[a, b]$. If $g^{\prime}(x) \neq 0, x \in(a, b)$, then there exists a function $F(x)$, $x \in[a, b]$, and a constant $d_{0}>0$ such that

$$
\int_{a}^{x} f(z) e^{i \omega g(z)} \mathrm{d} z=F(a)-F(x)+E(x)
$$

with $F(x)$ and $E(x)$ of the form

$$
\begin{aligned}
& F(x)=e^{i \omega g(x)} \int_{0}^{d_{0}} f\left(h_{x}(p)\right) e^{-\omega p} \frac{d h_{x}}{d p}(p) \mathrm{d} p, \\
& E(x)=e^{-\omega d_{0}} \int_{a}^{x} f(\kappa(z)) e^{i \omega g(z)} \frac{d \kappa}{d z}(z) \mathrm{d} z .
\end{aligned}
$$

Proof. We will prove the existence of decomposition (4.1) by the explicit construction of a new integration path for the integral. The construction of the path is illustrated in Figure 2. The first part of the new path is parameterized by $z=h_{a}(p)$, $p \in\left[0, d_{0}\right]$, such that $e^{i \omega g\left(h_{a}(p)\right)}=e^{i \omega g(a)} e^{-\omega p}$. This means that the parameterization $h_{a}(p)$ should satisfy $g\left(h_{a}(p)\right)=g(a)+i p$. The second part is parameterized by $z=\kappa(y), y \in[a, x]$, such that $g(\kappa(y))=g(y)+d_{0} i$. Finally, the last part is parameterized by $z=h_{x}(p), p \in\left[0, d_{0}\right]$, such that $g\left(h_{x}(p)\right)=g(x)+i p$. 


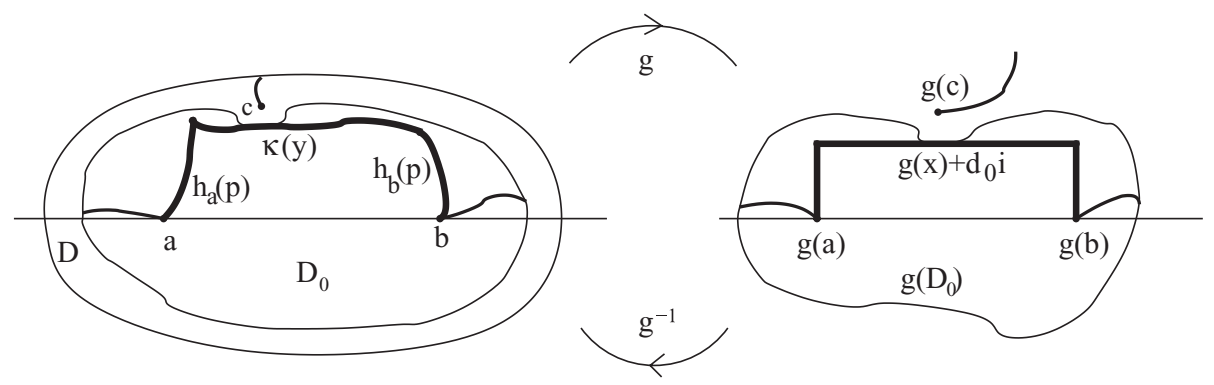

Figure 2. Illustration for Lemma 4.1 for the case where $g^{\prime}(a)=$ $g^{\prime}(b)=g^{\prime}(c)=0$. The figure shows the connection between the domain $D_{0}$ and its image $g\left(D_{0}\right)$.

Assuming that these paths exist and lie in $D$, we have by Cauchy's theorem

$$
\begin{aligned}
\int_{a}^{x} f(z) e^{i \omega g(z)} \mathrm{d} z=\int_{0}^{d_{0}} f\left(h_{a}(p)\right) e^{i \omega g\left(h_{a}(p)\right)} \frac{d h_{a}}{d p}(p) \mathrm{d} p \\
\quad+\int_{a}^{x} f(\kappa(y)) e^{i \omega g(\kappa(y))} \frac{d \kappa}{d y}(y) \mathrm{d} y-\int_{0}^{d_{0}} f\left(h_{x}(p)\right) e^{i \omega g\left(h_{x}(p)\right)} \frac{d h_{x}}{d p}(p) \mathrm{d} p .
\end{aligned}
$$

This decomposition has the form of (4.1).

It remains to show that such a path exists. Here, we will prove this is the case for an integration over $[a, b]$ with $g^{\prime}(a)=g^{\prime}(b)=0$. The easier case of an interval $[a, x]$ with no stationary points, or with a single stationary point at $a$, is proven along the same lines.

Since $g^{\prime}$ is analytic in $D$, any compact singly connected subset of $D$ will contain at most a finite number of isolated zeros of $g^{\prime}$. Since $g^{\prime}(x) \neq 0$ for $x \in(a, b)$, one can always construct such a subset $D_{0}$ with $[a, b] \subset \operatorname{int} D_{0}$, containing no zeros of $g^{\prime}$ except $a$ and $b$. Consider $g\left(D_{0}\right)$, with boundary $\partial g\left(D_{0}\right)$, and set $d_{0}$ to be the minimum vertical distance defined as

$$
d_{0}=\min \left\{\Im z \text { for } z \in \partial g\left(D_{0}\right) \cap i \mathbb{C}^{+} \text {satisfying } \Re z \in g([a, b])\right\} .
$$

Since $g$ is analytic and nonconstant, we have that the image of $[a, b]$ is strictly in the interior of the compact region $g\left(D_{0}\right)$. Hence, the minimum in (4.4) is well-defined and $d_{0}>0$.

The inverse of $g$ is typically a multivalued function on $g\left(D_{0}\right)$ with branch points at each point $g(z)$ where $g^{\prime}(z)=0$ [5]. In the present case those branch points are $g(a)$ and $g(b)$. The function $g$ can be made uniquely invertible by selecting arbitrary nonintersecting branch cuts connecting those points to $\partial g\left(D_{0}\right)$. These cuts can always be constructed in such a way that they do not intersect the rectangle. Define $g^{-1}$ as the branch that satisfies $g^{-1}(g(x))=x$ for $x \in[a, b]$. Then, the inverse of the rectangular path lies entirely within $D_{0}$, and hence, within the region of analyticity of $g$ and $f$.

A general decomposition in the presence of multiple stationary points can be obtained by repeatedly applying Lemma 4.1 on suitable subintervals. Note that the value of $d_{0}$ in the definition of $F$ and $E$ is determined by the size of $D_{0}$, or, more precisely, by the presence of stationary points $z \in \mathbb{C}$ that lie close to the 
interval $[a, b]$, and by the region of analyticity $D$ of $f$ and $g$. In most cases, $d_{0}$ may be quite large or even infinite.

The following theorem gives a decomposition for a one-dimensional integral with an $n$-dimensional integrand. A function in $n$ variables is called analytic if it is analytic in each variable. We denote such a function $f$ here by $f(\mathbf{x}, y)$, with $\mathbf{x} \in$ $\mathbb{C}^{n-1}$ and $y \in \mathbb{C}$. A similar notation is used for $g$.

Theorem 4.2. Assume $f$ and $g$ are $n$-dimensional functions that are analytic for $\mathbf{x}$ in an open complex neighourhood of a closed domain $B \subset \mathbb{R}^{n-1}$, and $y$ in an open complex neighbourhood $D(\mathbf{x})$ of $[a(\mathbf{x}), b(\mathbf{x})]$. If $\frac{\partial g}{\partial y}(\mathbf{x}, y) \neq 0$, for $\mathbf{x} \in B$, $a(\mathbf{x})<y<b(\mathbf{x})$, and if

$$
\begin{array}{lll}
\frac{\partial g}{\partial y}(\mathbf{x}, a(\mathbf{x})) \neq 0 & \text { or } \quad \frac{\partial g}{\partial y}(\mathbf{x}, a(\mathbf{x})) \equiv 0, & \forall \mathbf{x} \in B,
\end{array}
$$

then there exist functions $F$ and $E$, such that

$$
\int_{a(\mathbf{x})}^{b(\mathbf{x})} f(\mathbf{x}, y) e^{i \omega g(\mathbf{x}, y)} \mathrm{d} y=F(\mathbf{x}, a(\mathbf{x}))-F(\mathbf{x}, b(\mathbf{x}))+E(\mathbf{x}), \quad \forall x \in B,
$$

and with $F$ and $E$ of the form

$$
\begin{aligned}
F(\mathbf{x}, y) & =e^{i \omega g(\mathbf{x}, y)} \int_{0}^{d_{0}} f(\mathbf{x}, h(\mathbf{x}, p)) e^{-\omega p} \frac{\partial h}{\partial p}(\mathbf{x}, p) \mathrm{d} p, \\
E(\mathbf{x}) & =e^{-\omega d_{0}} \int_{a(\mathbf{x})}^{b(\mathbf{x})} f(\mathbf{x}, \kappa(\mathbf{x}, y)) e^{i \omega g(\mathbf{x}, y)} \frac{\partial \kappa}{\partial y}(\mathbf{x}, y) \mathrm{d} y,
\end{aligned}
$$

with $d_{0}>0$. The functions $F$ and $E$ are analytic in $\mathbf{x}$ in an open neighbourhood of $B$ if $a(\mathbf{x})$ and $b(\mathbf{x})$ are analytic.

Proof. For a fixed value of $\mathbf{x} \in B$, we can apply Lemma 4.1. This yields two functions of $y, F_{1}(y ; \mathbf{x})$ and $E_{1}(y ; \mathbf{x})$, such that

$$
\int_{a(\mathbf{x})}^{b(\mathbf{x})} f(\mathbf{x}, y) e^{i \omega g(\mathbf{x}, y)} \mathrm{d} y=F_{1}(a(\mathbf{x}) ; \mathbf{x})-F_{1}(b(\mathbf{x}) ; \mathbf{x})+E_{1}(b(\mathbf{x}) ; \mathbf{x}) .
$$

These functions can be identified with (4.8) and (4.9) by $F(\mathbf{x}, y):=F_{1}(y ; \mathbf{x})$ and $E(\mathbf{x}):=E_{1}(b(\mathbf{x}) ; \mathbf{x})$. However, as the constant $d_{0}(\mathbf{x})$ still depends on $\mathbf{x}$, it remains to be proven that it can be chosen independently of $\mathbf{x}$.

Recall that the region $D_{0}$ in the proof of Lemma 4.1 was chosen such that it contains no zeros of $g^{\prime}$, except possibly $a$ and $b$. The size of the region $D_{0}$ and, hence, of the constant $d_{0}$, is restricted only by the analyticity of $f$ and $g$, and by the presence of isolated stationary points other than $a$ and $b$. In the current multivariate application of the lemma, this means that $D_{0}(\mathbf{x})$ is chosen such that it contains no zeros of $\frac{\partial g}{\partial y}$, except possibly $(\mathbf{x}, a(\mathbf{x}))$ and $(\mathbf{x}, b(\mathbf{x}))$. Now consider a (complex) curve $c(\mathbf{x})$ of stationary points, i.e., $\frac{\partial g}{\partial y}(\mathbf{x}, c(\mathbf{x})) \equiv 0, \mathbf{x} \in B$. The value $d_{0}(\mathbf{x})$ could become arbitrarily small if $c(\mathbf{x})$ lies arbitrarily close to $[a(\mathbf{x}, b(\mathbf{x})]$. However, conditions (4.5) and (4.6), together with the closedness of $B$, guarantee that $c(\mathbf{x})$ either coincides with $a(\mathbf{x})$ or $b(\mathbf{x})$, or it is an isolated stationary point for all $\mathbf{x} \in B$ including $\mathbf{x} \in \partial B$. Therefore, $d_{0}(\mathbf{x})$ can be bounded from below by a constant $d_{0}>0$. 
Finally, we note that all factors in the expressions for $F$ and $E$ are analytic in $\mathbf{x}$, and the integral of an analytic function is again analytic if the integration boundaries are given by a constant, or by an analytic function [5]. Hence, $F(\mathbf{x}, a(\mathbf{x}))$, $F(\mathbf{x}, b(\mathbf{x}))$ and $E(\mathbf{x})$ are analytic in $\mathbf{x} \in B$ if the boundary functions $a(\mathbf{x})$ and $b(\mathbf{x})$ are analytic. By a similar reasoning as in the previous paragraph, $d_{0}$ can be chosen small enough, but still positive, such that $F$ and $E$ are analytic at least in an open complex neighbourhood of $B$.

Remark 4.3. Condition (4.5) requires that the boundary function $a(\mathbf{x})$ does not cross a curve $c(\mathbf{x})$ of stationary points in $y$ : either $a(\mathbf{x})$ and $c(\mathbf{x})$ are disjunct, or they coincide. If $a\left(\mathbf{x}_{1}\right)=c\left(\mathbf{x}_{1}\right)$ at a single point $\mathbf{x}_{1} \in B$, then the constant $d_{0}$ may become arbitrarily small. The function $F(\mathbf{x}, y)$ can still be shown to exist, but it may not be possible to evaluate the function using the path of steepest descent due to the presence of stationary points in the complex plane. Aside from the numerical singularity at such points, crossing a stationary point means that the line integral that connects the endpoints of the paths for $a$ and $b$ can no longer be discarded. Still, the function $F(\mathbf{x}, y)$ can be evaluated using any other path that yields exponential decay, as long as the total decomposition is justified by Cauchy's Theorem, and the integration path does not cross any stationary points. An example of this special case will be given in $\$ 6$.

We can now describe the total decomposition in the presence of stationary points. For $n$-dimensional functions, the equation $\frac{\partial f}{\partial y}(\mathbf{x}, y)=0$ has $(n-1)$-dimensional solutions $y=s_{i}(\mathbf{x}), i=1, \ldots, l$. As in the one-dimensional case, the integration region will be subdivided, using these solutions as new boundaries.

Theorem 4.4. Assume $f$ and $g$ are $n$-dimensional functions that are analytic for $\mathbf{x}$ in an open complex neighbourhood of a closed domain $B \subset \mathbb{R}^{n-1}$, and $y$ in an open complex neighbourhood $D(\mathbf{x})$ of $[a(\mathbf{x}), b(\mathbf{x})]$. Assume further that $\frac{\partial g}{\partial y}\left(\mathbf{x}, s_{i}(\mathbf{x})\right)=0$, $i=1, \ldots, l$, and $\frac{\partial g}{\partial y}(\mathbf{x}, y) \neq 0$ otherwise. If $s_{0}(\mathbf{x}):=a(\mathbf{x}) \leq s_{1}(\mathbf{x}) \leq \ldots \leq s_{l}(\mathbf{x}) \leq$ $s_{l+1}(\mathbf{x}):=b(\mathbf{x})$, and $a(\mathbf{x})$ and $b(\mathbf{x})$ satisfy (4.5)-(4.6), then there exist functions $F_{i}$ and $E_{i}$ of the form (4.8) and (4.9) such that

$$
\begin{aligned}
\int_{a(\mathbf{x})}^{b(\mathbf{x})} f(\mathbf{x}, y) e^{i \omega g(\mathbf{x}, y)} \mathrm{d} y= & \sum_{j=1}^{l+1}\left[F_{j}\left(\mathbf{x}, s_{j-1}(\mathbf{x})\right)-F_{j}\left(\mathbf{x}, s_{j}(\mathbf{x})\right)\right] \\
& +\sum_{j=1}^{l+1} E_{j}(\mathbf{x}), \quad \forall x \in B .
\end{aligned}
$$

Proof. We can write the integral as

$$
\int_{a(\mathbf{x})}^{b(\mathbf{x})} f(\mathbf{x}, y) e^{i \omega g(\mathbf{x}, y)} \mathrm{d} y=\int_{a(\mathbf{x})}^{s_{1}(\mathbf{x})} \cdot \mathrm{d} y+\int_{s_{1}(\mathbf{x})}^{s_{2}(\mathbf{x})} \cdot \mathrm{d} y+\ldots+\int_{s_{l}(\mathbf{x})}^{b(\mathbf{x})} \cdot \mathrm{d} y .
$$

The result follows from the repeated application of Theorem 4.2 .

4.2. Repeated one-dimensional integration. The results of the previous subsection can be used in a recursive setting in order to obtain a decomposition for an $n$-dimensional integral,

$$
I_{n}:=\int_{a_{1}}^{b_{1}} \int_{a_{2}\left(x_{1}\right)}^{b_{2}\left(x_{1}\right)} \int_{a_{3}\left(x_{1}, x_{2}\right)}^{b_{3}\left(x_{1}, x_{2}\right)} \ldots \int_{a_{n}\left(x_{1}, \ldots, x_{n}\right)}^{b_{n}\left(x_{1}, \ldots, x_{n}\right)} f(\mathbf{x}) e^{i \omega g(\mathbf{x})} \mathrm{d} x_{n} \mathrm{~d} x_{n-1} \ldots \mathrm{d} x_{1} .
$$


The decomposition of the inner integral in $x_{n}$ can be obtained by Theorem 4.4 Assume that the equation $\frac{\partial g}{\partial x_{n}}\left(\mathbf{x}, x_{n}\right)=0$ has $l$ solutions $x_{n}=s_{i}(\mathbf{x}), i=1, \ldots, l$. Then, the decomposition of the inner integral in $x_{n}$ has the form of (4.10). The functions $F_{i}\left(\mathbf{x}, s_{j}(\mathbf{x})\right)$ are analytic in $\mathbf{x}$, and have an oscillator of the form $g\left(\mathbf{x}, s_{j}(\mathbf{x})\right)$. Define $s_{0}(\mathbf{x})=a_{n}(\mathbf{x})$ and $s_{l+1}(\mathbf{x})=b_{n}(\mathbf{x})$; then every function $F_{j}$, for $j=$ $1, \ldots, l+1$, leads to two oscillators,

$$
\begin{aligned}
& g_{j, 1}(\mathbf{x}):=g\left(\mathbf{x}, s_{j-1}(\mathbf{x})\right), \\
& g_{j, 2}(\mathbf{x}):=g\left(\mathbf{x}, s_{j}(\mathbf{x})\right) .
\end{aligned}
$$

Obviously $g_{j, 1}(\mathbf{x})=g_{j-1,2}(\mathbf{x})$. These oscillators are $(n-1)$-dimensional analytic functions. The first index $i$ denotes the subinterval of $\left[a_{n}(\mathbf{x}), b_{n}(\mathbf{x})\right]$, the second index denotes an endpoint of that interval.

In the following we will denote an oscillator compactly by $g_{\lambda}$, where $\lambda$ is a multiindex. An integral corresponding to $g_{\lambda}$ can be decomposed again using Theorem 4.4 . If $g_{\lambda}\left(\mathbf{x}, x_{n-1}\right)$ has $l_{\lambda}$ stationary points $s_{\lambda, i}(\mathbf{x}), i=1, \ldots, l_{\lambda}$, this yields $l_{\lambda}+1$ functions $F_{\lambda, i}, i=1, \ldots, l_{\lambda}+1$. Denote by $s_{\lambda, 0}(\mathbf{x}):=a_{n-1}(\mathbf{x})$ and $s_{\lambda, l_{\lambda}+1}(\mathbf{x}):=$ $b_{n-1}(\mathbf{x})$. Each contribution has the form $F_{\lambda, i}\left(\mathbf{x}, s_{\lambda, i-1}(\mathbf{x})\right)$ or $F_{\lambda, i}\left(\mathbf{x}, s_{\lambda, i}(\mathbf{x})\right)$. The oscillators can be defined recursively by

$$
\begin{aligned}
& g_{\lambda, i, 1}(\mathbf{x}):=g_{\lambda}\left(\mathbf{x}, s_{\lambda, i-1}(\mathbf{x})\right), \\
& g_{\lambda, i, 2}(\mathbf{x}):=g_{\lambda}\left(\mathbf{x}, s_{\lambda, i}(\mathbf{x})\right) .
\end{aligned}
$$

These oscillators are $(n-2)$-dimensional analytic functions. The definitions can be extended recursively, applying Theorem 4.4 for each integration variable until the integral $I_{n}$ is fully written as a sum of integrals that are no longer oscillatory. Extending our notation, each recursive step adds two layers of indices to $\lambda$ : the decomposition of an integral with oscillator $g_{\lambda}$ yields the functions $F_{\lambda, i}$, $i=1, \ldots, l_{\lambda}+1$, and the evaluation of $F_{\lambda, i}$ in the endpoints leads to the new oscillators $g_{\lambda, i, 1}$ and $g_{\lambda, i, 2}$. After the final recursive step, we have functions $F_{\lambda^{\prime}}$ with $\operatorname{size}\left(\lambda^{\prime}\right)=2 n-1$, evaluated in points $x_{\lambda}$ with $\operatorname{size}(\lambda)=2 n$ of the form

$$
x_{\lambda}=g_{\lambda}(a)=\left(a, f_{1}(a), f_{2}\left(a, f_{1}(a)\right), f_{3}\left(a, f_{1}(a), f_{2}\left(a, f_{1}(a)\right)\right), \ldots\right),
$$

with $a \in\left[a_{1}, b_{1}\right]$. Examples will be given in $\$ 6$. The functions $f_{i}$ can either be one of the boundary functions $a_{j}$ or $b_{j}$ of $I_{n}$, or a curve of stationary points for one integration variable. In the following theorem, we use $F_{\lambda^{\prime}}$ to denote the function that is evaluated at $x_{\lambda}$ (i.e., $\lambda^{\prime}$ is $\lambda$ with the last index omitted).

Theorem 4.5. Assume $f$ and $g$ are $n$-dimensional functions that are analytic in a complex neighbourhood of the integration region of $I_{n}$, given by (4.11), with all boundary functions $a_{i}$ and $b_{i}$ analytic, $i=2, \ldots, n$. Define the functions $g_{\lambda}$ recursively by (4.12) - (4.13) and (4.14) - (4.15). If the following condition holds,

$$
\forall \lambda, \exists y: \frac{\partial g_{\lambda}}{\partial y}(\mathbf{x}, y) \neq 0,
$$

then there exist functions $F_{\lambda^{\prime}}$ and points $\mathbf{x}_{\lambda}$ such that

$$
I_{n}=\sum_{\operatorname{size}(\lambda)=2 n} s_{\lambda} F_{\lambda^{\prime}}\left(\mathbf{x}_{\lambda}\right)+O\left(e^{-\omega d_{0}}\right),
$$

with $s_{\lambda}= \pm 1$ and with a constant $d_{0}>0$. 
Proof. The construction of the functions $F_{\lambda}$ and the points $\mathbf{x}_{\lambda}$ follows from the recursive description given earlier in this section, based on applying Theorem 4.4 repeatedly for all integration variables. Condition (4.17) guarantees that each oscillator encountered for an integration variable $y$ is not independent of $y$. It remains to show in this proof that the error of the full decomposition decays exponentially fast as $O\left(e^{-\omega d_{0}}\right)$ with a constant $d_{0}>0$.

Consider the decomposition of the integration in $x_{n}$ of an $n$-dimensional oscillatory integrand, as given by Theorem 4.4. The error expression $E_{j}$ has the form of (4.9),

$$
E_{j}(\mathbf{x})=e^{-\omega d_{0, j}} \int_{a(\mathbf{x})}^{b(\mathbf{x})} f\left(\mathbf{x}, \kappa_{j}\left(\mathbf{x}, x_{n}\right)\right) e^{i \omega g\left(\mathbf{x}, x_{n}\right)} \frac{\partial \kappa_{j}}{\partial x_{n}}\left(\mathbf{x}, x_{n}\right) \mathrm{d} x_{n} .
$$

Function $f$ is analytic on a (finite) complex neighbourhood of the integration domain, and can therefore be bounded uniformly on that domain by a constant $M>0$. Additionally, we have $\left|e^{i \omega g\left(\mathbf{x}, x_{n}\right)}\right| \leq 1$ since $g\left(\mathbf{x}, x_{n}\right)$ is real. Finally, in order to bound the third factor $\frac{\partial \kappa_{j}}{\partial x_{n}}\left(\mathbf{x}, x_{n}\right)$, recall that $\kappa_{j}\left(\mathbf{x}, x_{n}\right):=g_{n}^{-1}\left(g\left(\mathbf{x}, x_{n}\right)+d_{0, j} i\right)$, where $g_{n}^{-1}(y)$ represents the inverse of $g$ with respect to $x_{n}$. We have

$$
\frac{\partial \kappa_{j}}{\partial x_{n}}\left(\mathbf{x}, x_{n}\right)=\frac{\partial g_{n}^{-1}}{\partial y}\left(g\left(\mathbf{x}, x_{n}\right)+d_{0, j} i\right) \frac{\partial g}{\partial x_{n}}\left(\mathbf{x}, x_{n}\right) .
$$

The derivative of $g$ is bounded, because $g$ is analytic on the (finite) integration domain. The derivative of $g_{n}^{-1}$ can only be unbounded if $g^{\prime}\left(\mathbf{x}, \kappa\left(\mathbf{x}, x_{n}\right)\right)=0$. This situation occurs when there is a stationary point along the path for the error integral. By construction, this is never the case. Hence, the third factor of (4.19) can also be bounded by a constant $N>0$. Combining these observations, we have

$$
\left|\int_{a_{1}}^{b_{1}} \int_{a_{2}\left(x_{1}\right)}^{b_{2}\left(x_{1}\right)} \int_{a_{3}\left(x_{1}, x_{2}\right)}^{b_{3}\left(x_{1}, x_{2}\right)} \ldots \int_{a_{n-1}(\mathbf{x})}^{b_{n-1}(\mathbf{x})} E_{j}(\mathbf{x}) \mathrm{d} x_{n-1} \ldots \mathrm{d} x_{1}\right| \leq D M N e^{-\omega d_{0, j}}
$$

with $D$ the size of the integration domain.

The decomposition for the integration in $x_{n}$ yields $l+1$ functions $F_{i}$, when there are $l$ stationary points $s_{i}(\mathbf{x})$ in $x_{n}$. From expression (4.8) for $F_{i}$, we see that each contribution to $I_{n}$ is of the form

$$
\int_{a_{1}}^{b_{1}} \int_{a_{2}\left(x_{1}\right)}^{b_{2}\left(x_{1}\right)} \int_{a_{3}\left(x_{1}, x_{2}\right)}^{b_{3}\left(x_{1}, x_{2}\right)} \cdots \int_{a_{n-1}(\mathbf{x})}^{b_{n-1}(\mathbf{x})} \tilde{f}(\mathbf{x}) e^{i \omega \tilde{g}(\mathbf{x})} \mathrm{d} \mathbf{x} .
$$

Each contribution has the form of $I_{n-1}$. The line of arguments can therefore be repeated in order to bound the error for the decomposition in $x_{n-1}$, and recursively for $x_{n-2}, \ldots, x_{1}$. The constant $d_{0}$ in (4.18) is obtained as the smallest of the $d_{0, j}$ constants.

Remark 4.6. Condition (4.17) explicitly excludes the case where $\frac{\partial g_{\lambda}}{\partial y}(\mathbf{x}, y) \equiv 0$. In that case, $g_{\lambda}(\mathbf{x}, y)=f(\mathbf{x})$ is independent of $y$, and hence, it is not an oscillator for the variable $y$. The corresponding integral cannot be decomposed. However, since the integral is not oscillatory, this case does not pose a problem: it can be evaluated using standard integration techniques. If $\frac{\partial g_{\lambda}}{\partial z}(\mathbf{x}, z, y) \neq 0$, the recursive procedure can be continued for the oscillatory integral in the variable $z$.

Remark 4.7. Throughout this section, we have assumed that equations of the form $\frac{\partial g}{\partial x_{n}}\left(\mathbf{x}, x_{n}\right)=0$ have $l$ solutions, where $l$ is a constant independent of $\mathbf{x}$. If $l$ 
depends on the value of $\mathbf{x}$, then the integration region can always be split into a number of regions where $l$ is a constant. This may introduce integrals for which conditions (4.5) and (4.6) in Theorem 4.2 cannot hold. Still, the decomposition can be computed following Remark 4.3. A numerical example of this special case will be given in $\$ 6$.

4.3. Integration on closed volumes. The procedure to locate the special points is simplified when the integration region is a closed and smooth $n$-dimensional volume without corner points. In order to see this, note that there are many equivalent ways of writing an integral over a closed and smooth volume in the general form of (4.11). In particular, the integration boundary functions $a_{i}$ and $b_{i}$ are not unique: they correspond to a certain parameterization of the volume, of which there are infinitely many. However, a different choice of integration boundary functions leads to a different set of critical points $x_{\lambda}$, as identified by the recursive procedure described in $\$ 4.2$. Although the resulting decomposition will be correct, we can expect that some of these points are merely an artifact of our arbitrary choice of boundary functions. Indeed, one can verify that such points appear twice in the decomposition, and that $\mathbf{x}_{\lambda}=\mathbf{x}_{\mu}, F_{\lambda^{\prime}}\left(\mathbf{x}_{\lambda}\right)=F_{\mu^{\prime}}\left(\mathbf{x}_{\mu}\right)$ and $s_{\lambda}=-s_{\mu}$. Hence, the artificial contributions cancel out. They need not be computed.

In [9], the nonresonance condition was formulated in order to avoid the presence of stationary points in one or more integration variables. The condition requires that $\nabla g(\mathbf{x})$ should not be orthogonal to the boundary of the integration region. For a smooth and closed integration region, the nonresonance condition can never be satisfied. In fact, the critical points are the points where $\nabla g=0$, and those points where the nonresonance condition is violated, i.e., where the gradient of the oscillator is orthogonal to the boundary. This was shown for a two-dimensional integral in $\$ 3.2$. We show the same holds for 3D. Consider a three-dimensional surface that can be represented locally by $\kappa(x, y)=\left[\begin{array}{ll}x & a(x, y)\end{array}\right]^{T}$ around a critical point $\mathbf{x}_{\lambda}$. The point is given by

$$
x_{\lambda}=g_{\lambda}\left(s_{1}\right)=\left(s_{1}, s_{2}\left(s_{1}\right), a\left(s_{1}, s_{2}\left(s_{1}\right)\right)\right),
$$

following (4.16). The function $s_{2}(x)$ and the value $s_{1}$ are found such that

$$
\begin{aligned}
& \left.\frac{\partial g(x, y, a(x, y))}{\partial y}\right|_{y=s_{2}(x)}=0 \\
& \left.\frac{\partial g\left(x, s_{2}(x), a\left(x, s_{2}(x)\right)\right)}{\partial x}\right|_{x=s_{1}}=0
\end{aligned}
$$

i.e., there is a stationary point in $x$ and $y$. The first relation leads to

$$
\frac{\partial g}{\partial y}+\frac{\partial g}{\partial z} \frac{\partial a}{\partial y}=0
$$

The second relation yields

$$
\begin{aligned}
& \frac{\partial g}{\partial x}+\frac{\partial g}{\partial y} \frac{\partial s_{2}}{\partial x}+\frac{\partial g}{\partial z}\left(\frac{\partial a}{\partial x}+\frac{\partial a}{\partial y} \frac{\partial s_{2}}{\partial x}\right)=\frac{\partial g}{\partial x}+\frac{\partial g}{\partial z} \frac{\partial a}{\partial x}+\frac{\partial s_{2}}{\partial x}\left(\frac{\partial g}{\partial y}+\frac{\partial g}{\partial z} \frac{\partial a}{\partial y}\right)=0 \\
& (4.21) \quad \Rightarrow \frac{\partial g}{\partial x}+\frac{\partial g}{\partial z} \frac{\partial a}{\partial x}=0 .
\end{aligned}
$$


Equations (4.20) and (4.21) together express the relations of orthogonality

$$
\begin{aligned}
& {\left[\frac{\partial g}{\partial x} \frac{\partial g}{\partial y} \frac{\partial g}{\partial z}\right]\left[\begin{array}{lll}
1 & 0 & \frac{\partial a}{\partial x}
\end{array}\right]^{T}=0} \\
& {\left[\frac{\partial g}{\partial x} \frac{\partial g}{\partial y} \frac{\partial g}{\partial z}\right]\left[\begin{array}{lll}
0 & 1 & \frac{\partial a}{\partial y}
\end{array}\right]^{T}=0}
\end{aligned}
$$

i.e., $\nabla g$ is orthogonal to $\kappa(x, y)$ at $\mathbf{x}_{\lambda}$.

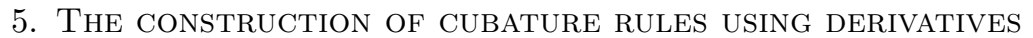

The decomposition of an oscillatory integral as described in $\$$ can be written as

$$
I[f]:=\int_{S} f(\mathbf{x}) e^{i \omega g(\mathbf{x})} \mathrm{d} \mathbf{x}=\sum_{\operatorname{size}(\lambda)=2 n} s_{\lambda} F_{\lambda^{\prime}}[f]\left(\mathbf{x}_{\lambda}\right)+O\left(e^{-\omega d_{0}}\right),
$$

with

$$
\begin{gathered}
F_{\lambda^{\prime}}[f]\left(\mathbf{x}_{\lambda}\right):=e^{i \omega g\left(\mathbf{x}_{\lambda}\right)} \int_{0}^{d_{0}} \ldots \int_{0}^{d_{0}} f\left(h_{1}\left(p_{1}\right), h_{2}\left(h_{1}\left(p_{1}\right), p_{2}\right), \ldots\right) e^{-\omega\left(\sum p_{i}\right)} \\
\frac{\partial h_{1}}{\partial p_{1}}\left(p_{1}\right) \frac{\partial h_{2}}{\partial p_{2}}\left(h_{1}\left(p_{1}\right), p_{2}\right) \ldots \frac{\partial h_{n}}{\partial p_{n}}\left(\ldots, h_{n-1}\left(\ldots, p_{n-1}\right), p_{n}\right) \mathrm{d} p_{1} \ldots \mathrm{d} p_{n} .
\end{gathered}
$$

The functions $h_{i}$ represent the optimal paths with respect to the oscillators that are implied by the multi-index $\lambda$. This is a generalization of the two-dimensional form given by (3.7). If the function $f$ is easily evaluated for complex arguments, tensor-product Gauss-Laguerre rules can be used to obtain an accurate approximation to each of the $F_{\lambda^{\prime}}[f]\left(\mathbf{x}_{\lambda}\right)$ values. This is a straightforward extension of Theorems 2.2 and 2.4. Alternatively, the function value $F_{\lambda^{\prime}}[f]\left(\mathbf{x}_{\lambda}\right)$ can be approximated by approximating $f$ locally around the point $\mathbf{x}_{\lambda}$. That is the approach taken in this section. The result is a cubature rule that requires only function values and derivatives of $f$ at $\mathbf{x}_{\lambda}$. The use of the tensor-product Gauss-Laguerre quadrature rule to evaluate the weights of the cubature rule will be illustrated in $\$ 6$.

5.1. A localised Filon's method. Filon's method for oscillatory integrals was already recalled for one-dimensional integrals in $\$ 2.3$. The multivariate extension is straightforward: if $f$ is approximated by a linear combination of basis functions, $f(\mathbf{x})=\sum_{i=1}^{N} a_{i} \phi_{i}(\mathbf{x})$, then $I[f]$ can be approximated by

$$
I[f] \approx Q[f]:=\sum_{i=1}^{N} w_{i} a_{i}, \quad \text { with } \quad w_{i}:=I\left[\phi_{i}\right] .
$$

In [7, 9] a polynomial basis is suggested, such that the value of $f$ and a number of its derivatives are interpolated in the critical points $\mathbf{x}_{\lambda}$. Depending on the number of critical points and the number of derivatives interpolated, the degree of the basis functions may have to be high. Owing to our decomposition of the integral into a sum of independent contributions however, the contributions can be approximated separately, i.e., there is no need for a global approximation of $f$. This will lead to a cubature rule with the same order of accuracy, but using a much lower degree of polynomials.

We will now construct an approximation for $F_{\lambda^{\prime}}[f]\left(\mathbf{x}_{\lambda}\right)$ as given in (5.2). Define the multi-index $i=i_{1} i_{2} \ldots i_{n}$ with $|i|=i_{1}+i_{2}+\ldots+i_{n}$, and denote 
$\left(x_{1}-y_{1}\right)^{i_{1}} \ldots\left(x_{n}-y_{n}\right)^{i_{n}}$ by $(\mathbf{x}-\mathbf{y})^{i}$. Then we can use the Taylor series of $f$ in the following way:

$$
F_{\lambda^{\prime}}[f]\left(\mathbf{x}_{\lambda}\right)=\sum_{|i| \leq \infty} \frac{F_{\lambda}\left[\left(\mathbf{x}-\mathbf{x}_{\lambda}\right)^{i}\right]\left(\mathbf{x}_{\lambda}\right)}{i_{1} ! i_{2} ! \ldots i_{n} !} f^{(i)}\left(\mathbf{x}_{\lambda}\right) .
$$

The truncated Taylor series can be used in order to obtain a convergent cubature rule for (5.3). Assume that the total order of the derivative at point $\mathbf{x}_{\lambda}$ is limited by $d_{\lambda}$; then we propose the cubature rule

$$
Q_{s}[f]:=\sum_{\operatorname{size}(\lambda)=2 n} \sum_{|i| \leq d_{\lambda}} w_{\lambda, i} f^{(i)}\left(\mathbf{x}_{\lambda}\right),
$$

with the weights given by

$$
w_{\lambda, i}:=s_{\lambda} \frac{F_{\lambda^{\prime}}\left[\left(\mathbf{x}-\mathbf{x}_{\lambda}\right)^{i}\right]\left(\mathbf{x}_{\lambda}\right)}{i_{1} ! i_{2} ! \ldots i_{n} !} .
$$

Remark 5.1. The method of constructing the cubature rule is as follows. The oscillator $g(\mathbf{x})$ and the integration domain $S$ determine the location of the critical points $\mathbf{x}_{\lambda}$ and the optimal paths, by the recursive procedure described in $₫$. Hence, the abscissae $\mathbf{x}_{\lambda}$ depend only on the oscillator and on the domain. The value of the weights is found by evaluating (5.6) along these paths. The weights depend in general on $\omega$. Finally, an approximation to $I[f]$ is obtained by evaluating the function $f$ and its derivatives in the abscissae and evaluating (5.5).

5.2. Convergence properties. In order to obtain the order of accuracy as a function of $\omega$ of the cubature rule (5.5), we will first examine the error in the truncation of (5.4). The size of the truncation error will determine the integration error.

Lemma 5.2. Consider the point $\mathbf{x}_{\lambda}$ with $\operatorname{size}(\lambda)=2 n$, and the oscillator $g_{\lambda}$ obtained by repeated one-dimensional integration. If the oscillator for integration variable $x_{j}$ has a stationary point of order $r_{j}, j=1, \ldots, n$, then we have, with $i$ the multi-index $i_{1} \ldots i_{n}$,

$$
\left|F_{\lambda^{\prime}}\left[\left(\mathbf{x}-\mathbf{x}_{\lambda}\right)^{i}\right]\left(\mathbf{x}_{\lambda}\right)\right|=O\left(\omega^{-\alpha_{\lambda, i}}\right), \quad \text { with } \quad \alpha_{\lambda, i}=\sum_{j=1}^{n} \frac{i_{j}+1}{r_{j}+1} .
$$

Proof. First consider a one-dimensional case. The general expression (5.2) for $F_{\lambda^{\prime}}$ simplifies to

$$
F_{\lambda^{\prime}}\left[\left(\mathbf{x}-\mathbf{x}_{\lambda}\right)^{i}\right]\left(\mathbf{x}_{\lambda}\right)=\int_{0}^{d_{0}}\left(h_{1}\left(p_{1}\right)-\mathbf{x}_{\lambda}\right)^{i} e^{i \omega g\left(h_{1}\left(p_{1}\right)\right)} \frac{d h_{1}}{d p_{1}}\left(p_{1}\right) \mathrm{d} p_{1},
$$

where $h_{1}\left(p_{1}\right)$ is found as the solution to $g\left(h_{1}\left(p_{1}\right)\right)=g\left(\mathbf{x}_{\lambda}\right)+p_{1} i$. Applying the transformation $q_{1}=\omega p_{1}$, this becomes

$$
F_{\lambda^{\prime}}\left[\left(\mathbf{x}-\mathbf{x}_{\lambda}\right)^{i}\right]\left(\mathbf{x}_{\lambda}\right)=\frac{e^{i \omega g\left(\mathbf{x}_{\lambda}\right)}}{\omega} \int_{0}^{d_{0} / \omega}\left(h_{1}\left(q_{1} / \omega\right)-\mathbf{x}_{\lambda}\right)^{i} e^{-q_{1}} \frac{d h_{1}}{d p_{1}}\left(q_{1} / \omega\right) \mathrm{d} q_{1} .
$$

Due to the exponential decay of the integrand, the asymptotic behaviour of the integral for large $\omega$ is given by the behaviour of the integrand near $q_{1}=0$.

Assume that the oscillator has a stationary point of order $r$. From the Taylor series of $g$,

$$
g(\mathbf{x})=g\left(\mathbf{x}_{\lambda}\right)+g^{(r+1)}\left(\mathbf{x}_{\lambda}\right) \frac{\left(\mathbf{x}-\mathbf{x}_{\lambda}\right)^{r+1}}{(r+1) !}+O\left(\left(\mathbf{x}-\mathbf{x}_{\lambda}\right)^{r+2}\right),
$$


we see that the parameterization of the optimal path behaves as

$$
h_{1}\left(p_{1}\right)=\mathbf{x}_{\lambda}+\sqrt[r+1]{\frac{(r+1) ! p_{1} i}{g^{(r+1)}\left(\mathbf{x}_{\lambda}\right)}}, \quad p_{1} \rightarrow 0 .
$$

Hence, we have $\left(h_{1}\left(q_{1} / \omega\right)-\mathbf{x}_{\lambda}\right)^{i} \sim \omega^{-i /(r+1)}$ and $\frac{d h_{1}}{d p_{1}}\left(q_{1} / \omega\right) \sim \omega^{1-1 /(r+1)}$. Adding these orders and the factor $\omega^{-1}$ in (5.7), we have

$$
\left|F_{\lambda^{\prime}}\left[\left(\mathbf{x}-\mathbf{x}_{\lambda}\right)^{i}\right]\left(\mathbf{x}_{\lambda}\right)\right|=O\left(\omega^{-\frac{i+1}{r+1}}\right) .
$$

In the multivariate case, this reasoning can be repeated for each integration variable. The resulting order is the sum of the orders corresponding to each integration variable. This leads to the result.

Theorem 5.3. The approximation of $I[f]$ by the cubature rule (5.5) has an error of the order

$$
I[f]-Q_{s}[f]=O\left(\omega^{-\alpha}\right), \quad \text { with } \quad \alpha=\min _{\operatorname{size}(\lambda)=2 n} \min _{|i|=d_{\lambda}+1} \alpha_{\lambda, i} .
$$

Proof. From Lemma 5.2, we see that the error in the truncation of (5.4) is asymptotically equivalent to the asymptotic order of the first discarded term. The latter is given by $\alpha_{\lambda, i}$ with $|i|=d_{\lambda}+1$. Hence, the order of the truncation error is found as the mimimum for all $\lambda$ and $i$ of $\alpha_{\lambda, i}$, with $|i|=d_{\lambda}+1$. The order of the error $I[f]-Q_{s}[f]$ is the same. The exponentially decaying error $e^{-\omega d_{0}}$ in (5.1) may be discarded because, asymptotically, it vanishes faster than any power of $\omega^{-1}$.

Remark 5.4. The convergence rate may actually be faster than the rate predicted by Theorem 5.3. This is due to the cancellation of moments at stationary points. In particular, it may be that $\mathbf{x}_{\lambda}=\mathbf{x}_{\mu}$, and that

$$
F_{\lambda^{\prime}}\left[\left(\mathbf{x}-\mathbf{x}_{\lambda}\right)^{i}\right]\left(\mathbf{x}_{\lambda}\right)-F_{\mu^{\prime}}\left[\left(\mathbf{x}-\mathbf{x}_{\mu}\right)^{i}\right]\left(\mathbf{x}_{\mu}\right)=o\left(\omega^{-\alpha_{\lambda, i}}\right)
$$

and $o\left(\omega^{-\alpha_{\mu, i}}\right)$, i.e., the difference of the moments at the special point $\mathbf{x}_{\lambda}$ can have lower order than the moments themselves.

\section{Numerical RESUlts}

In this section, we illustrate the convergence of the constructed cubature rules for some arbitrary functions $f$. The integration domains considered are the right half of a circle in $\$ 6.1$ the unit ball in 6.2 and a rectangular domain in $\$ 6.3$ and 86.4 We consider the Fourier oscillator and a more general oscillator that leads to an internal stationary point.

6.1. Half of a circle. We consider an integral over half a circle, written as

$$
I_{2}:=\int_{0}^{1} \int_{-\sqrt{1-x_{1}^{2}}}^{\sqrt{1-x_{1}^{2}}}\left(\cos \left(x_{1} x_{2}\right)+\frac{1}{2+x_{1}+x_{2}}\right) e^{i \omega\left(x_{1}+x_{2}\right)} \mathrm{d} x_{2} \mathrm{~d} x_{1} .
$$

The integration domain is shown in Figure 3. The set of critical points consists of the points $(0,-1)$ and $(0,1)$, because they are boundary points of the piecewise smooth integration domain, and the point $(\sqrt{2} / 2, \sqrt{2} / 2)$, because the gradient of the Fourier oscillator is orthogonal to the circle at that point. This also follows from the analysis following $\S 4$ we have

$$
\left\{\begin{array}{l}
g_{11}(x)=x-\sqrt{1-x^{2}} \\
g_{12}(x)=x+\sqrt{1-x^{2}}
\end{array}\right.
$$




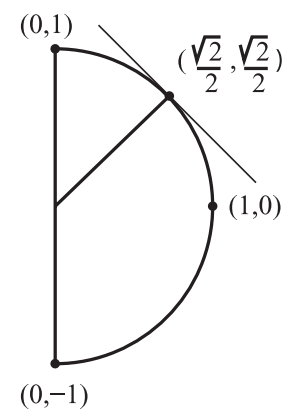

Figure 3. The integration domain for example 1. The gradient of the oscillator $\nabla g=\left[\begin{array}{ll}1 & 1\end{array}\right]^{T}$ is orthogonal to the tangent line at the point $(\sqrt{2} / 2, \sqrt{2} / 2)$.

with stationary points at $-\sqrt{2} / 2$, and $+\sqrt{2} / 2$ respectively. Since $x=-\sqrt{2} / 2$ lies outside the integration domain, the special points are

$$
\left\{\begin{array}{l}
\mathbf{x}_{1111}=(0,-1) \\
\mathbf{x}_{1112}=(1,0)
\end{array}\right.
$$

and

$$
\left\{\begin{array}{l}
\mathbf{x}_{1211}=(0,1) \\
\mathbf{x}_{1212}=(\sqrt{2} / 2, \sqrt{2} / 2) \\
\mathbf{x}_{1221}=(\sqrt{2} / 2, \sqrt{2} / 2) \\
\mathbf{x}_{1222}=(1,0)
\end{array}\right.
$$

This corresponds to the total decomposition

$$
\begin{aligned}
{\left[F_{111}\left(\mathbf{x}_{1111}\right)-F_{111}\left(\mathbf{x}_{1112}\right)\right] } & \\
- & {\left[F_{121}\left(\mathbf{x}_{1211}\right)-F_{121}\left(\mathbf{x}_{1212}\right)+F_{122}\left(\mathbf{x}_{1221}\right)-F_{122}\left(\mathbf{x}_{1222}\right)\right] . }
\end{aligned}
$$

The two contributions from the point $(1,0)$ cancel out, $F_{111}\left(\mathbf{x}_{1112}\right)=F_{122}\left(\mathbf{x}_{1222}\right)$; they are an artifact from the chosen parameterization of which the point appears to be a boundary point.

The moments $F_{121}\left[\left(\mathbf{x}-\mathbf{x}_{1212}\right)^{i}\right]\left(\mathbf{x}_{1212}\right)$ and $F_{122}\left[\left(\mathbf{x}-\mathbf{x}_{1221}\right)^{i}\right]\left(\mathbf{x}_{1221}\right)$ have a stationary point of order $r_{1}=1$ in the variable $x_{1}$, due to the stationary point $\sqrt{2} / 2$ of $g_{12}$; the other moments are regular. Using a fixed number of derivatives $d$ at each point, the moments at $(\sqrt{2} / 2, \sqrt{2} / 2)$ will asymptotically be the largest. From Lemma 5.2, the moment $F_{121}\left[\left(\mathbf{x}-\mathbf{x}_{1212}\right)^{i}\right]\left(\mathbf{x}_{1212}\right)$ with $i=(0, d)$ scales as $O\left(\omega^{-1-(d+1) / 2}\right)$. Hence, the first discarded moment with minimal order has order $\omega^{-1-(d+2) / 2}$. By Theorem [5.3 this is the leading order of the integration error. This is illustrated in Table 1. The columns with $d$ even have a higher convergence rate than predicted due to the (partial) cancellation of moments.

The total number of weights in the cubature formula for the rightmost column $(d=2)$ is 18: there are 3 critical points, and the evaluation of 6 partial derivatives with total order less than or equal to 2 is required in each point. The value of $I_{2}$ itself scales as the zero-th order moments, $\omega^{-3 / 2}$. Hence, the convergence rate of the relative error is 1.5 times smaller than the rate shown for the absolute error.

The weights were evaluated using tensor-product rules. Following [6, Rem.4.5], half-range Gauss-Hermite rules were used for evaluating one-dimensional integrals 
TABLE 1. Absolute error of the approximation of $I_{2}$ by a cubature rule using derivatives of maximal order $d$. The last row shows the value of $\log _{2}\left(e_{400} / e_{800}\right)$. The theoretically predicted asymptotic lower bound is shown between parentheses. The rules in columns $1-3$ require 3,9 and 18 function evaluations respectively.

\begin{tabular}{|c|ccc|}
\hline$\omega \backslash d$ & 0 & 1 & 2 \\
\hline 50 & $6.1 e-5$ & $1.4 e-5$ & $1.3 e-6$ \\
100 & $1.0 e-5$ & $2.6 e-6$ & $1.1 e-7$ \\
200 & $2.4 e-6$ & $4.5 e-7$ & $9.4 e-9$ \\
400 & $3.9 e-7$ & $7.9 e-8$ & $8.3 e-10$ \\
800 & $6.2 e-8$ & $1.4 e-8$ & $7.3 e-11$ \\
\hline rate & $2.7(2.0)$ & $2.5(2.5)$ & $3.5(3.0)$ \\
\hline
\end{tabular}

TABLE 2. Absolute error of the approximation of the zero-th order moment $F_{121}\left[\left(\mathbf{x}-\mathbf{x}_{1212}\right)^{0}\right]\left(\mathbf{x}_{1212}\right)$ by a tensor-product of GaussLaguerre and half-range Gauss-Hermite rules with $n$ points in each dimension. The last row shows the value of $\log _{2}\left(e_{100} / e_{50}\right)$. The theoretically predicted asymptotic lower bound is shown between parentheses.

\begin{tabular}{|c|ccc|}
\hline$\omega \backslash n$ & 1 & 2 & 3 \\
\hline 25 & $2.3 e-05$ & $4.6 e-08$ & $2.3 e-10$ \\
50 & $4.1 e-06$ & $4.1 e-09$ & $1.0 e-11$ \\
100 & $7.2 e-07$ & $3.6 e-10$ & $4.5 e-13$ \\
\hline rate & $2.5(2.5)$ & $3.5(3.5)$ & $4.5(4.5)$ \\
\hline
\end{tabular}

with a singularity due to a stationary point $[4$. Hence, we expect a convergence rate of the relative error of $O\left(\omega^{-n}\right)$, where $n$ is the number of quadrature rules used in each dimension. The absolute error scales as $O\left(\omega^{-n-3 / 2}\right)$. This is confirmed by the results in Table 2. Note that this approach is possible for more general $f$, and that the results require much less operations than the construction of the appropriate cubature rule. If applicable, and if high accuracy and efficiency is required, this approach is preferrable over the use of a cubature rule. For $\omega=100$ and $n=3,9$ function evaluations were required by the $2 \mathrm{D}$ tensor-product rule for an absolute error of $4.5 e-13$ and a relative error of $5.8 e-10$.

6.2. The unit ball. We consider an integral over the unit ball, written as

$$
I_{3}:=\int_{-1}^{1} \int_{-\sqrt{1-x_{1}^{2}}}^{\sqrt{1-x_{1}^{2}}} \int_{-\sqrt{1-x_{1}^{2}-x_{2}^{2}}}^{\sqrt{1-x_{1}^{2}-x_{2}^{2}}} e^{x_{1}+x_{2}^{2} x_{3}}\left(3 x_{3}+\cos \left(x_{2}\right)\right) e^{i \omega\left(x_{1}+x_{2}+x_{3}\right)} \mathrm{d} x_{3} \mathrm{~d} x_{2} \mathrm{~d} x_{1} .
$$

There are no corners in this example, since the integration domain is smooth everywhere. The critical points are those where the gradient of the Fourier oscillator is orthogonal to the boundary: $(-\sqrt{3} / 3,-\sqrt{3} / 3,-\sqrt{3} / 3)$ and $(\sqrt{3} / 3, \sqrt{3} / 3, \sqrt{3} / 3)$.

The decomposition for the integration variable $x_{3}$ yields the oscillators

$$
g_{11}\left(x_{1}, x_{2}\right)=x_{1}+x_{2}-\sqrt{1-x_{1}^{2}-x_{2}^{2}} \text { and } g_{12}\left(x_{1}, x_{2}\right)=x_{1}+x_{2}+\sqrt{1-x_{1}^{2}-x_{2}^{2}} \text {. }
$$


TABLE 3. Absolute error of the approximation of $I_{3}$ on the unit ball by a cubature rule using derivatives of maximal order $d$. The last row shows the value of $\log _{2}\left(e_{400} / e_{800}\right)$. The theoretically predicted asymptotic lower bound is shown between parentheses. The rules in columns $1-3$ require 2,8 and 20 function evaluations respectively.

\begin{tabular}{|c|ccc|}
\hline$\omega \backslash d$ & 0 & 1 & 2 \\
\hline 100 & $2.6 e-5$ & $2.4 e-6$ & $1.2 e-7$ \\
200 & $3.2 e-6$ & $3.6 e-7$ & $8.0 e-9$ \\
400 & $3.9 e-7$ & $5.2 e-8$ & $5.5 e-10$ \\
800 & $5.0 e-8$ & $3.8 e-9$ & $2.7 e-11$ \\
1600 & $6.3 e-9$ & $5.2 e-10$ & $1.7 e-12$ \\
\hline rate & $3.0(2.5)$ & $2.9(3.0)$ & $4.0(3.5)$ \\
\hline
\end{tabular}

These oscillators have a curve of stationary points in $x_{2}$, given by $x_{2}= \pm \sqrt{2-2 x_{1}^{2}}$. The relevant oscillators after the second decomposition are

$$
\begin{aligned}
& g_{1112}\left(x_{1}\right)=g_{1121}\left(x_{1}\right)=x_{1}-\sqrt{2-2 x_{1}^{2}}, \\
& g_{1212}\left(x_{1}\right)=g_{1221}\left(x_{1}\right)=x_{1}+\sqrt{2-2 x_{1}^{2}},
\end{aligned}
$$

with the stationary points $x_{1}= \pm \sqrt{3} / 3$.

At the point $(\sqrt{3} / 3, \sqrt{3} / 3, \sqrt{3} / 3)$, there is a stationary point of order 1 in $x_{1}$ and $x_{2}$. The size of the moments hence scales as $\omega^{-\left(i_{1}+1\right) / 2-\left(i_{2}+1\right) / 2-\left(i_{3}-1\right)}$. With the restriction $|i|=i_{1}+i_{2}+i_{3}=d+1$ from Theorem 5.3 , the leading order of the error is given by $\omega^{-(d+3) / 2-1}$. The rate is higher in the columns with $d$ even. The size of $I_{2}$ scales as the zero-th order moments, $\omega^{-1 / 2-1 / 2-1}=\omega^{-2}$. The convergence rate of the relative error is therefore 2 less than that shown for the absolute error.

Note that for $\omega=1600$ and $d=0$, only two function evaluations are required for an absolute error of $6.3 e-9$ and a relative error of $1.4 e-3$. The computation of the two weights in this case took less than a second of computation time. For comparison, a general purpose integration package was used on the same computer for the case $\omega=10$, using polar coordinates: it took 100, 000 function evaluations to obtain an absolute error of $1 e-7$. Assuming that the number of function evaluations scales at a cubic rate with respect to the frequency, due to the presence of oscillations in three dimensions, a comparable error for the case $\omega=1600$ would require roughly 400 billion function evaluations.

6.3. A rectangular domain with critical points. We consider a two-dimensional integral with the more general oscillator that was used in $\$ 3.3$.

$$
I_{2}:=\int_{0}^{0.5} \int_{0}^{1} \frac{1}{1+x+y} e^{i \omega\left(x^{2}-x y-y^{2}\right)} \mathrm{d} y \mathrm{~d} x .
$$

The stationary points in $x$ are given by $x=y / 2$, and the stationary points in $y$ are given by $y=-x / 2$. There is a critical point $(0,0)$ where $\nabla g=0$. The integration domain is illustrated in Figure 4. The contributions for this example integral come from the four corner points of the integration region: $(0,0),(0.5,0),(0.5,1)$ and 


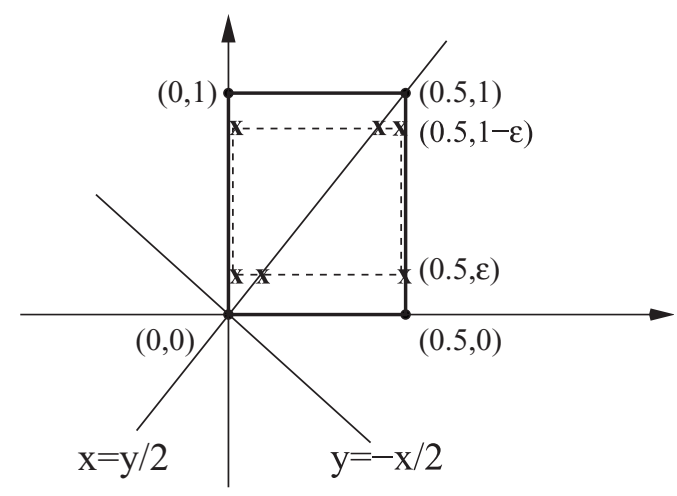

FiguRE 4. The points that contribute to the double integral $I_{2}$ for $g(x, y)=x^{2}-x y-y^{2}$ on the rectangle $[0,0.5] \times[0,1]$, indicated by the $\bullet$-s; the points that contribute to the integral over $[0,0.5] \times$ $[\epsilon, 1-\epsilon]$ are indicated by $x$-symbols.

$(0,1)$. The example was constructed however such that in the origin $(0,0)$ the conditions of Theorem 4.2 are not satisfied.

In order to find the decomposition, first consider the integral $I_{\epsilon}$ with the same integrand on $[0,0.5] \times[\epsilon, 1-\epsilon]$. The decomposition of $I_{\epsilon}$ consists of 8 contributions associated with the six points indicated by $x$-symbols in Figure 4 ,

$$
\begin{aligned}
I_{\epsilon} \sim & F_{111}(0, \epsilon)-F_{111}(\epsilon / 2, \epsilon)+F_{112}(\epsilon / 2, \epsilon) \\
& -F_{112}(0.5, \epsilon)-F_{121}(0,1-\epsilon)+F_{121}(0.5-\epsilon / 2,1-\epsilon) \\
& -F_{122}(0.5-\epsilon / 2,1-\epsilon)+F_{122}(0.5,1-\epsilon) .
\end{aligned}
$$

The first index $a$ in $F_{a b c}$ is the same for all contributions since it denotes the inverse of $g$ with respect to $y$, which is unique on the integration domain. The second index $b$ denotes $y=\epsilon$ when $b=1$, and $y=1-\epsilon$ when $b=2$. The third index denotes the two inverses of $g$ with respect to $x$, corresponding to the regions on the left and on the right of the line $x=y / 2$ respectively. In the limiting case $\epsilon \rightarrow 0$, we find that

$$
I_{2} \sim F_{112}(0,0)-F_{112}(0.5,0)-F_{121}(0,1)+F_{121}(0.5,1) .
$$

The example was constructed such that the decomposition of the inner integral in $y$ does not satisfy the conditions of Theorem 4.2. Indeed, the boundary function $y=0$ coincides with the curve of stationary points $y=-x / 2$ in exactly one point $x=0$. Following Remark 4.3 , we cannot evaluate the contribution $F_{112}(0,0)$ using the path of steepest descent for $y$ due to the presence of complex stationary points. However, an alternative integration path can be constructed. There are two disjunct regions where the integrand becomes arbitrarily small, corresponding to the two inverses of $g$ with respect to $y$. These regions can be characterized by $g_{1}^{-1}(x, y+i p)$ for $p>0, y \leq-x / 2$ and $g_{2}^{-1}(x, y+i p)$ for $p>0, y \geq-x / 2$. The integration path for $y$ at the point $(0.5,0)$ leads to the latter region. The integration path for $y$ at the point $(0,0)$ should therefore lead to the same region: the line integral that connects the paths can then be discarded. An equivalent condition is that the imaginary part of $g(x, y)$ should be positive along the total integration path for $y$, including 
TABLE 4. Absolute error of the approximation of $I_{2}$ by a cubature rule using derivatives of maximal order $d$. The last row shows the value of $\log _{2}\left(e_{400} / e_{800}\right)$. The theoretically predicted asymptotic lower bound is shown between parentheses. The rules in columns 1,2 and 3 require 4,12 and 24 function evaluations respectively.

\begin{tabular}{|c|ccc|}
\hline$\omega \backslash d$ & 0 & 1 & 2 \\
\hline 50 & $1.4 e-03$ & $1.6 e-04$ & $1.5 e-05$ \\
100 & $5.2 e-04$ & $4.0 e-05$ & $2.7 e-06$ \\
200 & $1.9 e-04$ & $1.0 e-05$ & $4.8 e-07$ \\
400 & $6.7 e-05$ & $2.6 e-06$ & $8.6 e-08$ \\
800 & $2.4 e-05$ & $6.5 e-07$ & $1.5 e-08$ \\
\hline rate & $1.48(1.5)$ & $1.99(2.0)$ & $2.49(2.5)$ \\
\hline
\end{tabular}

the discarded connecting part. For this particular example, we arbitrarily chose a linear path for $y$ from $y_{0}=0$ to the point $y_{1}=1-1 i$.

The results are shown in Table 4. Since $(0,0)$ is a stationary point for both integration variables, the absolute error is the largest for the contribution of the origin. The size of the first discarded term scales as $O\left(\omega^{(-d-3) / 2}\right)$ by Theorem 5.3 and hence this is also the size of the absolute error. The convergence rate of the relative error is 1 less than the rate shown for the absolute error.

\subsection{A degenerate critical point. Consider the integral}

$$
I_{d e g}:=\int_{-1}^{1} \int_{-1}^{1} \frac{1}{3+x+y} e^{i \omega\left(x^{3}+y^{3}\right)} \mathrm{d} y \mathrm{~d} x
$$

that has a degenerate stationary point of order 2 in both integration variables at the origin $(0,0)$. There are contributions from the four corner points $(-1,-1),(-1,1)$, $(1,-1)$ and $(1,1)$, and from the boundary points $(-1,0),(1,0),(0,-1)$ and $(0,1)$. The corresponding cubature rule uses function evaluations at these 9 critical points.

The leading order of the size of $I_{d e g}$ as a function of $\omega$ is determined by the contribution of the degenerate critical point $(0,0)$. Since $r=2$ for $x$ and for $y$, we expect that the value of $\left|I_{d e g}\right|$ behaves as $O\left(\omega^{-1 / 3} \omega^{-1 / 3}\right)=O\left(\omega^{-2 / 3}\right)$. The leading order of the error of the cubature rule behaves as $O\left(\omega^{-(2+d) / 3}\right)$. The theoretically predicted convergence rate is confirmed by the results in Table 5 .

\section{Concluding REMARKS}

A cubature rule was developed for the evaluation of oscillatory integrals of the form (1.1). The weights of the rules can be obtained efficiently by evaluating integrals with an integr and that decays exponentially fast in each integration variable; the higher the frequency, the faster the decay. The accuracy of the cubature likewise increases with the frequency.

The method is limited to analytic functions $g$ and a piecewise analytic integration domain. This is due to the application of Cauchy's Theorem from complex analysis. In the one-dimensional case, a similar restriction was lifted by approximating $g$ by its (analytic) truncated Taylor series. It was shown that this approximation leads to a convergent approach. Though it is reasonable to assume that this approach is extensible to the multivariate case, this is still the subject of further research. 
TABLE 5. Absolute error of the approximation of $I_{\text {deg }}$ by a cubature rule using derivatives of maximal order $d$. The last row shows the value of $\log _{2}\left(e_{400} / e_{800}\right)$. The theoretically predicted asymptotic lower bound is shown between parentheses. The rules in columns 1,2 and 3 require 9,27 and 54 function evaluations.

\begin{tabular}{|c|ccc|}
\hline$\omega \backslash d$ & 0 & 1 & 2 \\
\hline 50 & $5.3 e-03$ & $2.5 e-04$ & $2.9 e-05$ \\
100 & $2.7 e-03$ & $9.9 e-05$ & $9.0 e-06$ \\
200 & $1.3 e-03$ & $3.9 e-05$ & $2.8 e-06$ \\
400 & $6.7 e-04$ & $1.6 e-05$ & $8.9 e-07$ \\
800 & $3.4 e-04$ & $6.1 e-06$ & $2.9 e-07$ \\
\hline rate & $0.98(1.0)$ & $1.34(1.33)$ & $1.63(1.66)$ \\
\hline
\end{tabular}

\section{REFERENCES}

1. N. Bleistein and R. Handelsman. Asymptotic Expansions of Integrals. Holt, Rinehart and Winston, 1975.

2. P. J. Davis and P. Rabinowitz. Methods of Numerical Integration. Computer Science and Applied Mathematics. Academic Press Inc., 1984. MR760629 (86d:65004)

3. L. N. G. Filon. On a quadrature formula for trigonometric integrals, Proc. Roy. Soc. Edinburgh, 49:38-47, 1928.

4. W. Gautschi. Orthogonal Polynomials: Computation and Approximation. Clarendon Press, Oxford, 2004. MR2061539 (2005e:42001)

5. P. Henrici. Applied and Computational Complex Analysis Volume I. Wiley \& Sons, 1974. MR.0372162 (51:8378)

6. D. Huybrechs and S. Vandewalle. On the evaluation of highly oscillatory integrals by analytic continuation, SIAM J. Numer. Anal. 44 (2006), no. 3, 1026-1048. MR2231854

7. A. Iserles and S. P. Nørsett. Efficient quadrature of highly oscillatory integrals using derivatives, Proc. R. Soc. Lond. Ser. A Math. Phys. Eng. Sci., 461:1383-1399, 2005. MR2147752 (2006b:65030)

8. A. Iserles and S. P. Nørsett. On quadrature methods for highly oscillatory integrals and their implementation., BIT Numerical Mathematics 44, 4:755-772, 2005. MR2211043 (2006k:65060)

9. A. Iserles and S. P. Nørsett. Quadrature methods for multivariate highly oscillatory integrals using derivatives, Math. Comp. 75 (2006), no. 255, 1233-1258. MR2219027(2007e:65024)

10. A. Iserles and S. P. Nørsett. On the computation of highly oscillatory multivariate integrals with critical points. Technical Report NA08, University of Cambridge, 2005.

11. D. Levin. Fast integration of rapidly oscillatory functions, J. Comput. Appl. Math., 67(1):95101, 1996. MR1388139 (97a:65029)

12. S. Olver. Moment-free numerical integration of highly oscillatory functions, IMA J. Num. Anal., 26(2):213-227, 2006. MR2218631 (2006k:65064)

13. S. Olver. On the quadrature of multivariate highly oscillatory integrals over non-polytope domains, Numerische Mathematik 103 (2006), no. 4, 643-665. MR2221066

14. E. M. Stein. Harmonic analysis: Real-variable methods, orthogonality and oscillatory integrals. Princeton University Press, Princeton, New York, 1993. MR.1232192 (95c:42002)

15. R. Wong. Asymptotic approximation of integrals. SIAM, 2001. MR1851050 (2002f:41023)

Scientific Computing, Department of Computer Science, K.U.Leuven, Belgium E-mail address: daan.huybrechs@cs.kuleuven.be

Scientific Computing, Department of Computer Science, K.U.Leuven, Belgium

E-mail address: stefan.vandewalle@cs.kuleuven.be 